\title{
A Theoretical and Empirical Investigation of Feedback in Ideation Contests
}

\author{
Juncai Jiang \\ Pamplin School of Business, Virginia Tech, Blacksburg, Virginia 24061, USA, jcjiang@vt.edu \\ Yu Wang* \\ College of Business, California State University, Long Beach, Long Beach, California 90840, USA, yu.wang@csulb.edu
}

\begin{abstract}
T deation contests are commonly used across public and private sectors to generate new ideas for solving problems, cre1 ating designs, and improving products or processes. In such a contest, a firm or an organization (the seeker) outsources an ideation task online to a distributed population of independent agents (solvers) in the form of an open call. Solvers compete to exert efforts and the one with the best solution wins a bounty. In evaluating solutions, the seeker typically has subjective taste that is unobservable to solvers. In practice, the seeker often provides solvers with feedback, which discloses useful information about her private taste. In this study, we develop a game-theoretic model of feedback in unblind ideation contests, where solvers' solutions and the seeker's feedback are publicly visible by all. We show that feedback plays an informative role in mitigating the information asymmetry between the seeker and solvers, thereby inducing solvers to exert more efforts in the contest. We also show that some key contest and solver characteristics (CSC, including contest reward, contest duration, solver expertise, and solver population) have a direct effect on solver effort. Interestingly, by endogenizing the seeker's feedback decision, we find that the optimal feedback volume increases with contest reward, contest duration, solver expertise, but decreases with solver population. Thus, CSC elements also have an indirect effect on solvers' effort level, with feedback volume mediating this effect. Employing a dataset from Zhubajie.com, a leading online ideation platform in China, we find empirical evidence that is consistent with these theoretical predictions.
\end{abstract}

Key words: ideation contest; feedback; information asymmetry; crowdsourcing; contest and solver characteristics History: Received: January 2019; Accepted: October 2019 by Fred Feinberg, after 2 revisions.

\section{Introduction}

In today's world, businesses across various industries need to innovate and evolve faster than ever before to stay ahead of the competition. Researchers and practitioners have placed an increasing focus on ideationthe process of generating new ideas and solutions for business problems, creating designs, and improving goods, services or processes. In the past, employees and contractors were the principal sources of new ideas. However, in-house ideation can be costly, subject to free riding, and limited by the number and scope of ideas (Luo and Toubia 2015, Toubia 2006). Recent technological advances have enabled firms and organizations (i.e., seekers) to outsource ideation projects on the Internet to a distributed group of experts and enthusiasts (i.e., solvers) in the form of an open call, or "crowdsourcing" (Bayus 2013, Boudreau and Lakhani 2013, Howe 2006). In particular, ideation contest has gained massive popularity where the crowdsourcing process is organized as an open contest with a bounty for the best ideas (Prpić et al. 2015, Terwiesch and Ulrich 2009). As compared to traditional methods, ideation contest can be exceptionally effective in generating more and better solutions at a lower cost from a large number of solvers with diverse knowledge and expertise (Gupta et al. 2019, Jiang et al. 2018, Poetz and Schreier 2012).

For marketing professionals, ideation contests offer an easy, practical and cost-effective method to tap into large online communities for creative ideas and outof-the-box solutions. They are able to crowdsource concepts for the next-generation products, designs of new landmarks and buildings, logo designs for brands and events, and many more. For instance, in its annual contest "Crash the Super Bowl," Frito-Lay invites consumers to create commercials for Doritos and promises at least one entry to be aired during the Super Bowl. From 2006 to 2016, Frito-Lay received over 36,000 entries, which helped the company win the first place on the USA Today Ad Meter Poll a number of times. AT\&T, Coca-Cola, and American Express all sponsor online creativity contests to seek innovative ideas for the company (Rathi 2014). As another example, Threadless has built its entire business model on ideation contests. Every week, it receives about a thousand designs from its online community of artists. Ten of them are selected as 
winning designs, which are then used for making clothing and other products. The winning artists receive a reward as well as royalties for sales. Whereas large companies are able to host ideation contests independently, smaller firms and organizations often resort to established online ideation platforms, such as InnoCentive, 99Designs, Zhubajie, and IdeaConnection, that help connect seekers with millions of potential solvers.

Despite its popularity, ideation contest suffers from information asymmetry between the seeker and solvers. This is because ideation contests typically involve broad and non-detailed tasks where relevant parties have their own private and subjective taste for potential solutions (Boudreau et al. 2011, Prpić et al. 2015, Terwiesch and Xu 2008). Although the seeker determines the winning solution based on her preferences, solvers do not have full information about them. Consider a company running an ideation contest for a new logo. The seeker-the top executive or a group of decision-makers in the company-may have preferences for the color, font, style, shape, and size of the logo, or any combination of the above. Yet, solvers participating in the contest may not fully comprehend the subtlety in the logo design. "Does the seeker like a modern or retro feel, a dynamic or static image, a masculine or feminine look?" More broadly speaking, logos subtly convey brand personality and positioning-something the seeker understands but cannot fully articulate.

Interestingly, during the course of the contest, the seeker often provides solvers with feedback comments that contain useful information about her private taste. To the best of our knowledge, there is scant research on seekers' feedback even though it is a commonly seen feature across many online ideation platforms. To fill this gap, we ask three important research questions in the context of ideation contests. First, how does the seeker's feedback volume affect solvers' effort level? Second, what is the impact of contest and solver characteristics (CSC) on solver efforts? In this study, we focus our attention on four CSC elements: contest reward, contest duration, solver expertise, and solver population. Last but not the least, by endogenizing the feedback volume decision of the seeker, we are able to examine how CSC elements influence feedback volume. Specifically, we are interested in understanding whether feedback functions as a mediating variable in the relationship between CSC and solvers' effort level. The answer to the last research question is central to understanding the underlying process through which CSC elements affect solvers' effort level and has important implications on the design of ideation contests.

To address these research questions, we develop a game-theoretic model of ideation contest with feedback, in which a number of solvers compete to win a pre-specified amount of reward. The seeker selects the winner based on solvers' performance, which in turn depends on their effort and the match value between the seeker and solvers. This match value term captures the information asymmetry due to the private taste of the seeker. That is, while the seeker has perfect information about her own taste, solvers do not but have some belief about it. The seeker can provide feedback that discloses information on her taste. Feedback is modeled as a noisy signal that allows solvers to update their belief following Bayesian learning, thus resulting in greater match value. In this model, while solvers choose how much effort to expend, the seeker decides the optimal feedback volume. Consistent with our empirical context, ideation contest is "unblind" in the sense that both solutions submitted by solvers and feedback provided by the seeker are publicly visible to all (Bockstedt et al. 2016, Wooten and Ulrich 2015, 2017).

Our theoretical results reveal an informative role of feedback: feedback mitigates the information asymmetry between the seeker and solvers, thereby inducing solvers to expend greater effort. We also identify a direct effect of CSC in that solvers' optimal effort level increases with contest reward, contest duration and solver expertise, but decreases with solver population. By endogenizing the seeker's feedback strategy, we show that the seeker's optimal feedback volume depends on CSC. Specifically, the optimal feedback volume increases with contest reward, contest duration, and solver expertise, but decreases with solver population. Thus, our analysis uncovers an indirect effect CSC elements have on solvers' effort through feedback volume, indicating that the seeker's feedback plays a mediating role in the relationship between CSC and solvers' effort. We collect data from 9771 logo design contests hosted on Zhubajie.com, the leading ideation platform in China. We find empirical evidence that is consistent with our theoretical predictions in the context of logo design contests.

In addition, our key theoretical findings are invariant when model assumptions are relaxed to allow for endogenous solver entry and pre-feedback solver efforts. Various robustness tests also indicate that our empirical results are insensitive to alternative definitions and operationalization of key variables.

Our contribution to the literature is fourfold. First, we contribute to the marketing literature on new product innovation and idea generation (e.g., Luo and Toubia 2015, Stephen et al. 2016, Toubia 2006) by modeling and empirically analyzing online ideation contests with feedback, a relatively new yet promising idea generation process that has been understudied in the marketing field. Second, while information asymmetry between the seeker and solvers is an 
inherent feature of ideation contests, to the best of our knowledge, we make the first attempt to investigate the use of feedback to mitigate information asymmetry. Our analytical model provides theoretical grounds for the informative role of feedback, which is validated empirically using the dataset we collected from Zhubajie.com. Thus, our theory and empirical investigation complement prior literature by providing a new explanation for the use of feedback in ideation contests. ${ }^{1}$ Third, this study complements the previous literature on the optimal design of ideation contests by simultaneously examining four CSC variables. Although prior research has investigated contest reward (Gupta et al. 2019, Liu et al. 2014, Slot 2013) and solver population (Boudreau et al. 2011, Terwiesch and $\mathrm{Xu} 2008$ ), contest duration and solver expertise are largely ignored. Our work fills this gap in the literature. Lastly, we endogenize the seeker's feedback decision to reveal that the seeker chooses feedback volume strategically depending on CSC. Thus, feedback plays a partial mediating role in the relationship between CSC and solver effort. This finding is further validated through empirical analysis. As such, we contribute to the previous studies on online feedback since they unanimously take feedback as exogenous (e.g., Wooten and Ulrich 2017).

The remainder of this study is organized as follows. Section 2 discusses the related literature. Section 3 presents the theoretical model and solution, from which we develop our hypotheses. Section 4 empirically tests the hypotheses. In section 5, we examine the robustness of our main results. We present the conclusions, limitations and future research directions in section 6 .

\section{Literature Review}

Our research draws on two streams of literature. We see our paper contributing to the marketing literature on ideation. Marketing practitioners and researchers have long recognized the importance of ideation, as it is essential to the "design and marketing of new products, to marketing strategy, and to the creation of effective advertising copy" (Toubia 2006). Early research on ideation mostly focuses on the in-house idea generation and selection process (e.g., Burroughs et al. 2011, Rossiter and Lilien 1994, Toubia 2006, Toubia and Florés 2007). Later works examine firm practice that takes a direction to involve external ideators on online platforms (see, e.g., Bayus 2013, Chan et al. 2015, Luo and Toubia 2015, Stephen et al. 2016). Bayus (2013) investigates Dell's IdeaStorm forum where users, who are mainly Dell customers, propose ideas and Dell decides whether to implement them. He finds that users with past success are less likely to continue proposing good ideas, while comments from other users can effectively mitigate this negative effect. Using similar data collected from Dell's IdeaStorm, Chan et al. (2015) reveal how peer-to-peer and peer-to-firm interactions influence users' subsequent idea generation. Luo and Toubia (2015) find that on online idea generation platforms the task structure has contrasting effects on users with different levels of knowledge-high-knowledge users prefer abstract cues such as problem decomposition while lowknowledge users do better with concrete cues such as stimulus ideas. In a series of experiments, Stephen et al. (2016) show that high connectivity of users in online ideation platforms can lead to low innovativeness, as connected users tend to come up with similar ideas and receive similar or redundant inspirations from one another. The context of these studies is different from ours because they focus on platforms where non-contest ideation processes take place. We extend this literature stream by explicitly modeling ideation contests in which multiple solvers compete to provide the best solution. ${ }^{2,3}$ We also empirically test the role of feedback in these ideation contests.

This study also draws on the emerging literature that investigates various issues in ideation contests: information asymmetry, feedback, and contest and solver characteristics. Terwiesch and $\mathrm{Xu}$ (2008) are among the first to formally propose a model of ideation contests. They identify information asymmetry as the key difference between ideation contests and other types of contests (e.g., expertise-based contests and trial-and-error contests). They suggest that "the seeker's taste, which is uncertain for the solver, plays an important role in determining what constitutes a good solution" and "the performance of a solution has a significant noise term that reflects heterogeneity of solvers' solutions in matching the seeker's taste." Building on Terwiesch and Xu (2008), Boudreau et al. (2011) further show that information asymmetry can greatly influence market outcomes using a dataset from Topcoder. However, neither paper explicitly explores the possibility that seekers can be strategic in using feedback comments to reduce information asymmetry, a question we address.

Despite its prevalence, the use of feedback in the context of ideation contests has largely been ignored. ${ }^{4}$ A notable exception is Wooten and Ulrich (2017) who ran a field experiment on performance feedback (in the form of ratings) in ideation contests. Specifically, they compare three alternative feedback policies: a directed feedback policy that gives feedback related to solvers' performance during the contest, a random feedback policy that leaves completely random feedback, and a no feedback policy where no feedback is provided at all. They show that, compared with the other two policies, the directed feedback policy induces solver participation and reduce quality 
variation as the contest progresses. Moreover, directed feedback policy could result in lower top-end quality of ideation solutions, although the average quality is unambiguously higher. Instead of ratings, we study text comments that reveal the seeker's taste in ideation solutions. We show that feedback comments can effectively reduce the information asymmetry inherent in ideation contests and lower solvers' risk of investing efforts. In addition, Wooten and Ulrich (2017) take feedback as an exogenous process through explicit manipulation in the experimental design. In contrast, we allow the seeker to make strategic decisions on feedback volume. We find both theoretically and empirically that seekers are indeed strategic in choosing feedback volume depending on the CSC elements of the contests.

Our study also adds to the nascent but growing literature on the optimal design of ideation contests. This literature stream examines how contest characteristics, such as solver population and contest reward affect the output of ideation contests. In an analytical model, Terwiesch and Xu (2008) show that although a larger solver population can reduce solver effort by intensifying competition, open entry can still be an optimal strategy for the seeker if the primary concern is the maximum performance from the crowd, because a large solver population can increase solver diversity and improve the output of the contest. Subsequently, Boudreau et al. (2011) analyze data from Topcoder and find that a larger solver population reduces solvers' average performance but has no significant impact on the maximum performance, providing empirical support for Terwiesch and $\mathrm{Xu}$ (2008). In a field experiment conducted on Taskcn.com, Liu et al. (2014) find that a higher contest reward can induce solvers to submit more solutions of higher quality. Hofstetter et al. (2018) demonstrate that winning a contest and receiving a reward can positively influence solver participation in subsequent contests. Based on this result, they recommend that the contest platform should encourage multiple reward levels in ideation contests. Terwiesch and $\mathrm{Xu}$ (2008) reveal that a performance-contingent reward regime can more effectively motivate solvers to expend their effort than a fixed-price reward regime. Jiang et al. (2018) suggest that instant cash rewards could lower the quality of solutions by drawing a large number of unqualified solvers into the contest. Gupta et al. (2019) cast doubt on the use of ideation contests in user content generation by showing that monetary reward may reduce the amount of non-monetary incentives (such as "likes" and "upvotes") awarded by peers for social recognition, due to the "taint" effect. Slot (2013) examines the size, number, and spread of rewards on the creativity (the degree of originality, unusualness, and novelty) of solvers in online idea generation contests. She finds that solvers' creativity increases with the size and number of rewards but decreases with the spread of rewards. We extend this literature stream by simultaneously examining the impact of CSC elements (solver population, contest reward, contest duration, and solver expertise) on solver effort, as well as the mediating role of feedback in the relationship between CSC and solvers' effort level.

\section{Theory and Hypothesis Development}

Consider an unblind ideation contest initiated by a seeker in which there are $N(N \geq 2)$ homogenous solvers (indexed by $i$ ). Following the contests and tournaments literature (e.g., Aoyagi 2010, Ederer 2010, Terwiesch and Xu 2008), solver performance can be measured in a one-dimensional space. We model the ideation contest as a single-winner contest. The seeker selects the solver with the highest performance and offers a pre-specified reward $V$, while the remaining solvers receive zero payoff. This single-winner allocation rule is the most popular format in ideation contests (Terwiesch and $\mathrm{Xu}$ 2008).

The game unfolds in two stages. In the first stage, the seeker determines her feedback volume. In the second stage, all solvers simultaneously choose how much effort to put into the contest. The sequence of moves reflects the fact that solvers' effort decisions are more flexible than the seeker's feedback volume decision, that is, solvers can always decide whether to expend more effort after the seeker gives feedback and the volume of feedback is known. ${ }^{5}$ We employ the subgame perfect Nash equilibrium and solve the model by backward induction. Model notations are summarized in Table A1 in Online Appendix A.

\subsection{Stage Two: Solvers' Effort Level}

Solvers are risk neutral and exogenously endowed with expertise $a$. Solver $i$ 's performance when expending effort level $e_{i}$ is given by the following:

$$
d_{i}=a e_{i}+\mu_{i} .
$$

The first term takes the multiplicative form, which implies that the marginal return of effort on performance increases with solver expertise (Ederer 2010, Moldovanu and Sela 2001). ${ }^{6}$ The second term captures the match value that is determined by how well solver i's solution appeals to the seeker's taste. Before receiving feedback, solver $i$ 's match value is a random draw from a normal distribution with a mean of $\mu_{\text {prior }}$ and a variance of $\tau^{2}$ : 


$$
\mu_{i} \sim N\left(\mu_{\text {prior }}, \tau^{2}\right)
$$

Feedback comments are conceptualized as noisy signals that contain information about the seeker's private taste, which can help solvers improve the match value of their solutions. Suppose that the seeker provides a total of $M$ feedback signals, $\mathbf{X}=\left\{x_{m}\right\}_{m=1, \ldots . M}$, which are observed by all solvers because the contest is "unblind" in nature. We assume that all feedback signals are i.i.d. drawn from a normal distribution centered around $\mu_{\text {feedback, }}$ given by the following:

$$
x_{m} \sim N\left(\mu_{\text {feedback }}, \sigma^{2}\right) .
$$

We assume $\mu_{\text {feedback }}>\mu_{\text {prior }}$ to capture the fact that feedback contains useful information about the seeker's taste. $\sigma^{2}$ is the variance and $1 / \sigma^{2}$ measures the precision of feedback signals. We assume that solvers follow Bayesian learning; that is, they learn from feedback signals $\mathbf{X}=\left\{x_{m}\right\}_{m=1, \ldots, M}$ such that their match values are updated following the Bayes' rule. This approach provides a parsimonious yet flexible framework for modeling the effect of feedback on solvers' performance. Thus, upon receiving $M$ feedback signals, solver i's posterior match value follows the following distribution:

$$
\mu_{i} \mid \mathbf{X} \sim N\left(\frac{M \tau^{2}}{M \tau^{2}+\sigma^{2}} \bar{x}+\frac{\sigma^{2}}{M \tau^{2}+\sigma^{2}} \mu_{\text {prior }}, \frac{\tau^{2} \sigma^{2}}{M \tau^{2}+\sigma^{2}}\right),
$$

where $\bar{x}=\frac{1}{M} \sum_{m=1}^{M} x_{m}$ is the sample mean. From Equation (4), we observe that feedback has two separate effects on solvers' match value: a matchenhancing effect and an uncertainty-reduction effect. The match-enhancing effect occurs because the posterior mean of the match value $E\left[\frac{M \tau^{2}}{M \tau^{2}+\sigma^{2}} \bar{x}+\frac{\sigma^{2}}{M \tau^{2}+\sigma^{2}} \mu_{\text {prior }}\right]=$ $\frac{M \tau^{2}}{M \tau^{2}+\sigma^{2}} \mu_{\text {feedback }}+\frac{\sigma^{2}}{M \tau^{2}+\sigma^{2}} \mu_{\text {prior }}$ improves and increasingly approaches $\mu_{\text {feedback }}$ as feedback volume $M$ increases. The uncertainty-reduction effect emerges as the variance of the posterior match value $\frac{\tau^{2} \sigma^{2}}{M \tau^{2}+\sigma^{2}}$ decreases with $M$, which implies that solvers' posterior match value becomes less dispersed when they receive more feedback signals. In other words, solvers are more certain about the seeker's taste when feedback volume is higher.

Equation (4) indicates that, after receiving feedback signals, solvers' performance follows a normal distribution given by the following:

$d_{i} \mid \mathbf{X} \sim N\left(a e_{i}+\frac{M \tau^{2}}{M \tau^{2}+\sigma^{2}} \bar{x}+\frac{\sigma^{2}}{M \tau^{2}+\sigma^{2}} \mu_{\text {prior }}, \frac{\tau^{2} \sigma^{2}}{M \tau^{2}+\sigma^{2}}\right)$.
Thus, the difference in performance between solvers $i$ and $k$ is also normally distributed:

$$
d_{i}-d_{k} \mid \mathbf{X} \sim N\left(a\left(e_{i}-e_{k}\right), \frac{2 \tau^{2} \sigma^{2}}{M \tau^{2}+\sigma^{2}}\right) .
$$

When comparing two solvers' performance, we find from Equation (6) that the match-enhancing effect of feedback is cancelled out because they received the same feedback signals, but the uncertainty-reduction effect remains. Consequently, the probability that solver $i$ achieves a higher level of performance than solver $k$ is given by the following:

$$
\operatorname{Pr}\left(d_{i}>d_{k} \mid \mathbf{X}\right)=\Phi\left(\frac{a\left(e_{i}-e_{k}\right)\left(M \tau^{2}+\sigma^{2}\right)}{2 \tau^{2} \sigma^{2}}\right),
$$

where $\Phi(\cdot)$ is the c.d.f. of a standard normal distribution. Thus, solver $i$ 's winning probability is given by the following:

$$
\begin{aligned}
\operatorname{Pr}\left(w_{i n}\right) & =\prod_{k \neq i} \operatorname{Pr}\left(d_{i}>d_{k} \mid \mathbf{X}\right) \\
& =\prod_{k \neq i} \Phi\left(\frac{a\left(e_{i}-e_{k}\right)\left(M \tau^{2}+\sigma^{2}\right)}{2 \tau^{2} \sigma^{2}}\right) .
\end{aligned}
$$

We assume that solvers have identical utility functions that are separable with respect to the benefit expected from the contest and the cost of the effort exerted. Solver $i$ 's expected benefit from participating in the contest, $V \operatorname{Pr}\left(w i n_{i}\right)$, increases with contest reward and winning probability. We assume a quadratic cost function of effort $\alpha(T) e_{i}{ }^{2}$, where $\alpha(T)$ is the cost sensitivity of effort and is a function of contest duration $T . \alpha(T)$ is assumed to be sufficiently large to guarantee the concavity of the utility function, or equivalently, $\alpha(T)>\bar{\alpha}$, where $\bar{\alpha}$ is a function of model parameters and is defined in Online Appendix B. In line with the prior literature (e.g., Zhang 2016), we assume that the marginal cost of effort decreases with contest duration, $\alpha^{\prime}(T)<0$. The intuition of this assumption is that when the contest duration is longer, solvers are less likely to face time pressure and time constraints, so the marginal cost of effort becomes lower.

As such, in stage two, each solver trades off his effort for the likelihood of winning the reward by choosing the effort level that maximizes the expected utility:

$$
\max _{e_{i}} U\left(e_{i}\right)=V \operatorname{Pr}\left(\operatorname{win}_{i}\right)-\alpha(T) e_{i}{ }^{2}
$$

We focus on symmetric equilibrium in the sense that symmetric solvers choose the same effort level. The solvers' optimal effort level is characterized in Proposition 1. 
Proposition 1. There is a unique symmetric pure-strategy Nash equilibrium in solvers' effort level:

$$
e^{*}=\frac{a V(N-1)\left(M \tau^{2}+\sigma^{2}\right)}{2^{N-\frac{1}{2}} \sqrt{\pi} \alpha(T) \tau^{2} \sigma^{2}} .
$$

The equilibrium effort level increases with feedback volume, contest reward, contest duration, and solver expertise, but decreases with solver population.

Proof. See Online Appendix B.

Proposition 1 indicates that solvers expend greater efforts when they receive more feedback comments from the seeker. This result can be attributed to the uncertainty-reduction effect of feedback. As feedback volume increases, solvers become more certain about the seeker's taste and the extent of information asymmetry between the seeker and solvers is mitigated. With more precise information, solvers can better assess their performance relative to those of others and hence their likelihood of winning. As a result, solvers strategically invest greater efforts into the contest. We call this the informative role of feedback. Proposition 1 also identifies a direct effect of CSC on solvers' effort level. The benefit of exerting effort is higher when contest reward increases. Therefore, solvers expend more effort when reward is higher. Longer contest duration reduces the marginal cost of effort and hence incentivizes solvers to exert more effort. Higher solver expertise boosts the marginal return of effort on performance and thus induces solvers to expend greater effort. In addition, an increase in solver population intensifies competition among solvers, thereby decreasing their winning probability. Thus, solvers strategically reduce effort when solver population increases.

\subsection{Stage One: Seeker's Feedback Strategy}

In this subsection, we return to stage one, in which the seeker rationally anticipates solvers' behavior when choosing her feedback volume to maximize profit given by:

$$
\max _{M} \Pi=\mathrm{E}\left[\max _{i}\left\{d_{i}\right\}\right]-V-\beta M .
$$

The first term is the expected maximum performance of all solvers, which is the expected benefit that the seeker obtains from the contest. At the same time, the seeker incurs costs from two sources. First, the seeker needs to pay the winner a contest reward of $V$. Second, composing and sending feedback is costly. We assume a linear cost function of $\beta M$. To guarantee the existence of an interior solution, we innocuously assume that $\bar{\beta}>\beta>\beta$, where both $\bar{\beta}$ and $\beta$ are functions of the model parameters specified in Online Appendix B. In Proposition 2, we characterize the seeker's optimal feedback volume.

Proposition 2. The seeker's optimal feedback volume is given by:

$$
M^{*}=\frac{\sigma}{\tau} \sqrt{\frac{\mu_{\text {feedback }}-\mu_{\text {prior }}-\tau^{2} \int_{-\infty}^{\infty} z d \Phi(z)^{N}}{\beta-\frac{a^{2} V(N-1)}{2^{N+\frac{1}{2}} \sqrt{\pi} \alpha(T) \sigma^{2}}}}-\frac{\sigma^{2}}{\tau^{2}} .
$$

The equilibrium feedback volume increases with contest reward, contest duration, and solver expertise, but decreases with solver population.

Proof. See Online Appendix B.

This proposition can be explained by how CSC elements impact the uncertainty-reduction effect of feedback. Higher contest reward magnifies the uncertainty-reduction effect and, as a result, the marginal benefit of providing feedback comments is greater. Recognizing this, the seeker strategically increases feedback volume. Similarly, the uncertainty-reduction effect and thus the marginal benefit of offering feedback are enhanced when contest duration is longer, solver expertise is higher, and solver population is smaller. Propositions 1 and 2 together imply CSC's indirect effect-through feedback volume-on solvers' effort level. In other words, the relationship between CSC and solvers' effort level is partially mediated by feedback volume. Table 1 summarizes our key model predictions from Propositions 1 and 2.

\subsection{Hypotheses Development}

Our theoretical framework generates three sets of hypotheses that are amenable to empirical testing. Drawn from Proposition 1, Hypothesis 1 is concerned with how feedback volume affects solvers' effort level; Hypothesis 2 lays out the direct effect of CSC on solvers' effort level. Hypothesis 3 is derived from Proposition 2 and addresses how CSC elements affect feedback volume.

\section{Table 1 Summary of Key Model Predictions}

\begin{tabular}{lcc}
\hline Variable & Solvers' effort level & The Seeker's feedback volume \\
\hline Feedback Volume & $\uparrow$ & n/a \\
Contest Reward & $\uparrow$ & $\uparrow$ \\
Contest Duration & $\uparrow$ & $\uparrow$ \\
Solver Expertise & $\uparrow$ & $\uparrow$ \\
Solver Population & $\downarrow$ & $\downarrow$ \\
\hline
\end{tabular}


Hypothesis 1. (H1). The Effect of Feedback Volume on Effort: Solvers' efforts are higher in ideation contests with greater feedback volume.

Hypothesis 2. (H2). The Direct Effects of Contest and Solver Characteristics on Effort:

H2(a). Solvers' efforts are higher in ideation contests with higher rewards.

H2(b). Solvers' efforts are higher in ideation contests with longer duration.

H2(c). Solvers' efforts are higher in ideation contests with higher solver expertise.

H2(d). Solvers' efforts are lower in ideation contests with larger solver population.

Hypothesis 3. (H3). The Effects of Contest and Solver Characteristics on Feedback Volume:

H3(a). The seeker provides more feedback when contest reward is higher.

H3(b). The seeker provides more feedback when contest duration is longer.

H3(c). The seeker provides more feedback when solver expertise is higher.
H3(d). The seeker provides less feedback when the solver population is larger.

Hypotheses 1-3 are summarized in Figure $1 .^{7}$

\section{Empirical Investigation}

\subsection{Research Context}

Our empirical setting is Zhubajie.com, a leading ideation contest website in China founded in 2006. As of December 2015, Zhubajie had hosted over 5 million contests, attracted 13.6 million solvers, and given out nearly US\$3 billion in rewards. It is the world's largest online marketplace for ideation contests in terms of the size of the solver pool. ${ }^{8}$ Registration on Zhubajie is free for both seekers and solvers, and there is no fee for solvers to participate in contests. Zhubajie collects $20 \%$ of contest reward as its commission and distributes the remaining $80 \%$ to the winner-a revenue model similar to those of other platforms.

Zhubajie offers services in many categories. Of these, graphic/logo design, marketing/sales, and web design are the top three in terms of popularity. We focus on the logo design category for the following reasons. First, because logo design is a type of ideation project, information asymmetry arising from the seeker's private taste is prominent (Terwiesch and

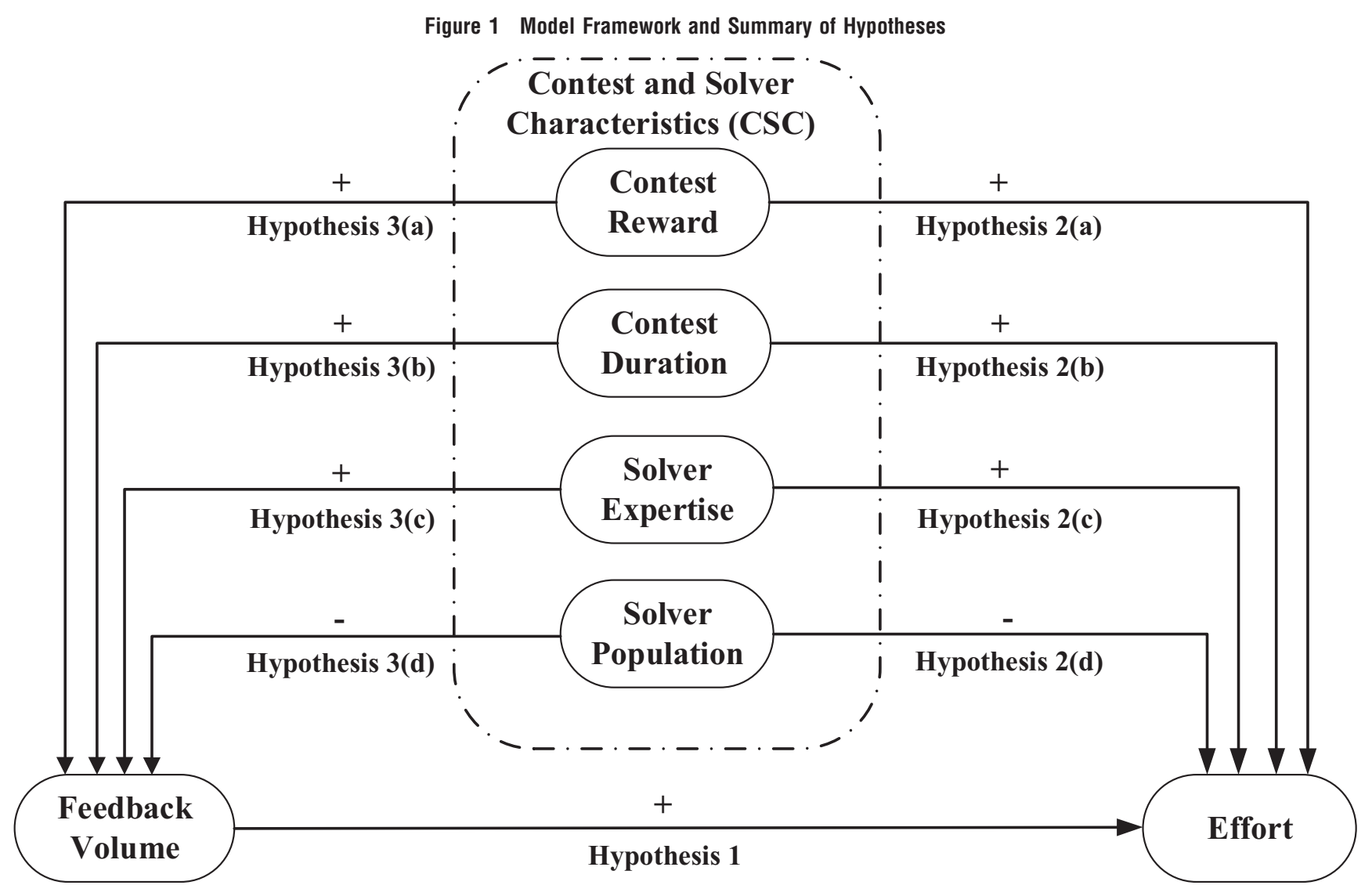


$\mathrm{Xu}$ 2008). Second, the company or brand logo is one of the most important business assets lying at the core of business branding strategies. Third, logo design requires considerable effort from solvers, so they tend to strategically choose their effort level when facing competition. Lastly, logo design contests display large variations in CSC, hence providing a rich context for empirical analysis.

A logo design contest begins when a seeker posts an announcement of the task on the platform. Figure 2 provides an example from Witmart.com, Zhubajie's US portal. AArealty, the seeker in this example, created a logo design contest with a US\$200 reward, a 30-day duration, and a description stating the task's purpose, requirements and basic information about the company. Then, solvers could register and begin submitting solutions. During the time period covered by our data (between March 2007 and March 2010), Zhubajie adopted an unblind format where all submitted logo designs and seeker feedback comments were viewable by all contestants. This practice is consistent with our theoretical model setup. Figure 3 shows an example of AArealty's feedback about a solution, which can be accessed by all solvers. At the conclusion of the contest, the seeker selected the winner who then received the reward.

\subsection{Data Description}

We collected information relevant to our study from all 11,584 logo design contests hosted by Zhubajie between March 2007 and March 2010. We first dropped incomplete contests and those with more than one winner, which comprised nearly $8 \%$ of the contests in our sample. The major reason for multiple winners was that when a seeker failed to select a winner within the allotted time frame, the platform would step in and evenly distribute the reward to all participating solvers. Next, we eliminated contests in which only one solver participated because the solver might not have acted strategically when there was no competition. Lastly, we removed contests with a reward below 50 Chinese yuan (CNY). ${ }^{9}$ Most of these contests were launched as test runs and thus failed to attract solvers. The resulting dataset included a sample of 9771 logo design contests in which 415,779 solvers submitted a total of 572,046 logo designs and won nearly 3.4 million CNY in rewards. An average logo contest in our data offered a reward of 346.55 CNY, had a duration of 10.72 days, attracted 54.51 solvers, included 6.70 feedback comments, and received 57.24 solutions.

\subsection{Variables}

Effort. On the solver side, our key dependent variable is the effort level. Since solver effort is not directly observable, we measure it by Number of Solutions, defined as the total number of logo designs a solver submitted in the course of the contest. In general, a larger number of solutions indicate higher effort level exerted by the solver. ${ }^{10}$ Number of Solutions is then averaged over all solvers in the contest to obtain contest-level measure of solver effort.

Figure 2 Example of a Logo Design Contest [Color figure can be viewed at wileyonlinelibrary.com]

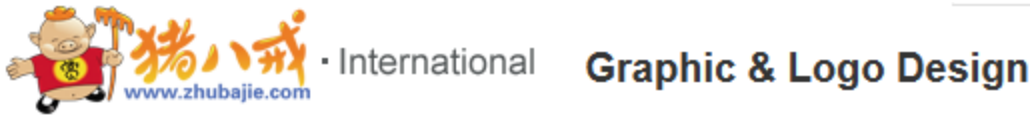

Home How lt Works Portfolio Customer Testimonials Post a Cont:

\title{
Logo Design for a Realtor watchlist \\ Employer: AARealty 娄 0 | Job ID: 35 | Category: Graphic \& Logo Design | Job type: Contest
}

\author{
Job Status: Completed ? \\ Bidding Started: Jul 09, 2011 11:50 AM \\ Job Reward: US\$200.00 \\ Bidding Ended: Aug 08, 2011 11:50 AM
}

\section{Description}

I need a logo for our realty company. The company name is AARealty, can separate the AA and Realty or Combine. Our company specilizes in commercial real estate, and high-end residential. I want the design to be used in letterheads, envelops, receipts, cups, cars, flags, and sellers displays. Please check with our website www.har.com/aarealty for information about our company. 
Feedback Volume. We define feedback as the text comments sent to solvers by the seeker regarding her taste (such as preferences for the colors, style, font, and size of the logo). Two independent coders read each comment and judged whether it contained information about the seeker's taste. Intercoder agreement was $92 \%$, suggesting high consistency in identifying seeker feedback. Where disagreements existed, they were resolved by the authors. Feedback Volume for each contest is the total number of text comments from the seeker that contained taste information.

Contest Reward. Contest reward is the monetary incentive offered by the seeker to the winner. We express the reward amount in CNY.

Contest Duration. The duration of the contest is calculated as the difference between its end and start dates expressed in the number of days. ${ }^{11}$

Solver Population. Solver population is the total number of solvers who participated in the contest.

Solver Expertise. Prior research used various paradigms to assess expertise, including prior knowledge, task-specific skills, and acquired experience (Winch 2010). We employed Average Income as the measure of solver expertise, because a solver's track record is a strong indicator of his knowledge, skills and experience. Specifically, Average Income is obtained by dividing a solver's cumulative income earned from the ideation platform (in CNY) by the total number of contests in which he participated. Then, we compute the average over all solvers in the contest to obtain the contest-level measure of solver expertise.

Feedback-Seeking Volume. The feedback literature in psychology and organizational behavior has long recognized that recipients do not passively wait for feedback. Instead, they proactively request feedback from the provider, thereby engaging in the so-called "feedback-seeking behavior" (for a review, see Ashford et al. 2003). In our research context, solvers seek feedback on Zhubajie through text messages with explicit requests. Two independent coders read the solvers' text messages to identify feedback-seeking behavior. Intercoder agreement was $88 \%$, suggesting high consistency in identifying solvers' feedback-seeking behavior. Where there were disagreements, the authors resolved them. For each

Figure 3 Example of Feedback Related to Logo Design [Color figure can be viewed at wileyonlinelibrary.com]

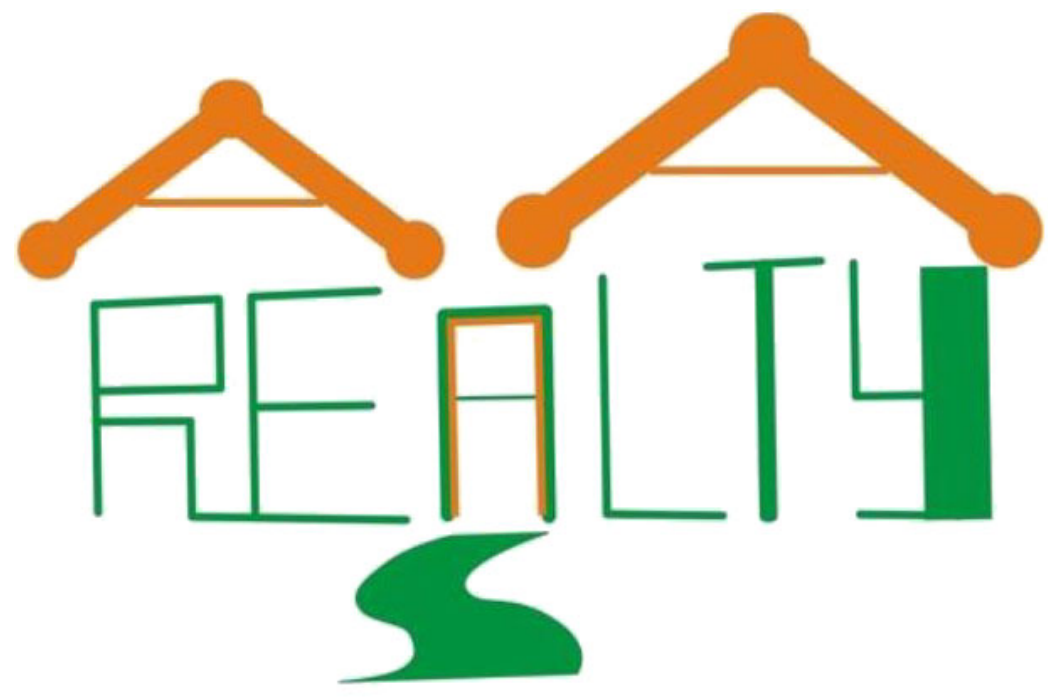

AARealty

This logo is very creative but is a little bit too busy in my opinion. I want the character to be easier to recognize and the style could be more serious. Also, I do not like the orange and green color. Can you use more blue colors? 
contest, feedback-seeking volume was measured by the total number of feedback-seeking text messages from solvers. Although feedback-seeking volume was not considered in our analytical model, we used it as an instrument variable in the empirical analysis.

Description Length. Description Length is the total number of words (i.e., Chinese characters) in the task description. We use it as an instrumental variable to tackle the potential endogeneity problem.

Table 2 summarizes the descriptive statistics and the correlation matrix of these variables.

\subsection{Empirical Analysis}

We now turn to the empirical framework to test the three sets of hypotheses established in section 3 . Since empirical analysis is conducted at the contest level, we use subscript $j$ for contests. We propose the following specification to assess how feedback and CSC affect the solvers' effort level:

$$
\begin{aligned}
\ln \left(\text { Effort }_{j}+1\right) & =\beta_{0}+\beta_{1} \ln \left({\text { Feedback } \left.\text { Volume }_{j}+1\right)}+{ }_{2} \ln \left(\text { Contest Reward }_{j}\right)\right. \\
& +\beta_{3} \ln \left(\text { Solver Expertise }_{j}+1\right) \\
& +\beta_{4} \ln \left(\text { Solver Population }_{j}\right) \\
& +\beta_{5} \ln \left({\text { Contest } \left.\text { Duration }_{j}\right)}\right)+\varepsilon_{j} .
\end{aligned}
$$

Similarly, to assess the effect of CSC on feedback volume, we develop the following econometric model:

$$
\begin{aligned}
\ln \left(\text { Feedback Volume }_{j}+1\right) & =\beta_{6}+\beta_{7} \ln \left(\text { Contest Reward }_{j}\right) \\
& +\beta_{8} \ln \left(\text { Solver Expertise }_{j}+1\right) \\
& +\beta_{9} \ln \left({\text { Solver } \left.\text { Population }_{j}\right)}\right. \\
& +\beta_{10} \ln \left({\text { Contest } \left.\text { Duration }_{j}\right)}\right) \\
& +v_{j} .
\end{aligned}
$$

We add one to their value and then take the natural log for variables that could take the value of zero (e.g., Effort, Solver Expertise and Feedback Volume), while performing the log-transformation for other variables. This method facilitates the interpretation of our results. In Equations (13) and (14), $\varepsilon_{j}$ and $v_{j}$ include all unobservable factors that affect solvers' effort level in contest $j$ and seeker $j$ 's feedback volume, respectively. Additionally, both $\varepsilon_{j}$ and $v_{j}$ are normally distributed.

If $\varepsilon_{j}$ and $v_{j}$ were independent of each other, we would be able to separately estimate the two regression models in Equations (13) and (14). However, there may be unobservable heterogeneity among contests in the error terms that simultaneously affects the solvers' choices of effort and the seeker's decision about feedback volume. In other words, $\varepsilon_{j}$ and $v_{j}$ are likely to be correlated. Consequently, we must address two econometric issues in our specifications. A trivial issue is that separate estimations of Equations (13) and (14) lead to efficiency loss in parameter estimates, which can be easily solved by using seemingly unrelated regression (SUR). Moreover, we should also account for a simultaneity problem in Equation (13) because Feedback Volume is endogenously determined, leading to biased estimates (Greene 2000). ${ }^{12}$

We pursue the instrumental variable (IV) approach to correct the endogeneity bias. Valid instruments should correlate with the seeker's decision on feedback volume and affect the solvers' effort level through feedback volume only. Our first instrument

\begin{tabular}{|c|c|c|c|c|c|c|c|c|c|c|c|c|c|c|c|}
\hline Variable & Mean & $S D$ & Min & $\operatorname{Max}$ & (1) & (2) & (3) & (4) & (5) & (6) & (7) & (8) & (9) & (10) & (11) \\
\hline (1) Number of Solutions & 1.05 & 0.25 & 0.13 & 5.92 & 1 & & & & & & & & & & \\
\hline (2) Elapsed Time & 3.69 & 6.03 & 0.02 & 299.76 & 0.13 & 1 & & & & & & & & & \\
\hline (3) Feedback Volume & 6.70 & 13.31 & 0 & 409 & 0.31 & 0.07 & 1 & & & & & & & & \\
\hline (4) Contest Reward & 346.55 & 464.43 & 50 & 10,000 & 0.05 & 0.20 & 0.19 & 1 & & & & & & & \\
\hline (5) Contest Duration & 10.72 & 12.46 & 1 & 376 & 0.07 & 0.87 & 0.09 & 0.28 & 1 & & & & & & \\
\hline (6) Solver Population & 54.51 & 47.51 & 2 & 1060 & 0.12 & 0.17 & 0.24 & 0.63 & 0.24 & 1 & & & & & \\
\hline (7) Average Income & 95.29 & 54.36 & 0 & 428.96 & 0.09 & 0.13 & 0.08 & 0.35 & 0.10 & 0.21 & 1 & & & & \\
\hline (8) Tenure & 3.49 & 1.73 & 0 & 12.57 & 0.03 & 0.04 & 0.01 & 0.03 & -0.03 & -0.02 & 0.69 & 1 & & & \\
\hline (9) Experience & 274.37 & 237.93 & 0 & 3647 & -0.12 & -0.05 & -0.05 & -0.12 & -0.07 & -0.14 & 0.21 & 0.58 & 1 & & \\
\hline $\begin{array}{l}\text { (10) Feedback-Seeking } \\
\text { Volume }\end{array}$ & 7.18 & 18.02 & 0 & 930 & 0.45 & 0.17 & 0.44 & 0.38 & 0.18 & 0.57 & 0.08 & -0.05 & -0.13 & 1 & \\
\hline (11) Description Length & 63.98 & 26.92 & 0 & 173 & -0.02 & -0.09 & -0.09 & 0.50 & 0.20 & 0.36 & 0.31 & 0.05 & -0.08 & 0.12 & 1 \\
\hline
\end{tabular}
is Feedback-Seeking Volume. It has long been shown that feedback-seeking behavior has a direct and positive impact on feedback volume (Ashford and Cummings 1983, Ashford and Tsui 1991). At the same time, feedback-seeking behavior is unlikely to be directly correlated with solvers' effort level, since there is no obvious link between them. Our second instrument is Description Length. Contest description

Table 2 Summary Statistics and Correlation Matrix 
is another way for the seeker to provide contestrelated information, including her private taste. We expect that a lengthy (vs. short) contest description contains more information about the seeker's preferences; therefore, there is less need for utilizing feedback. Stated differently, Description Length is negatively related to Feedback Volume. At the same time, Description Length has no direct effect on solvers' effort level. Thus, these two instruments can help us control for unobserved contest and solver characteristics that affect solver effort and feedback volume at the same time. Therefore, Feedback-Seeking Volume and Description Length satisfy the exclusion restriction and are appropriate instruments. We add Feedback-Seeking Volume and Description Length to Equation (14) as independent variables, as follows:

$$
\begin{aligned}
& \ln \left(\text { Feedback }_{\text {Volume }}+1\right)=\beta_{6}+\beta_{7} \ln \left(\text { Contest Reward }_{j}\right) \\
&+\beta_{8} \ln \left({\text { Contest } \left.\text { Duration }_{j}\right)}\right) \\
&+\beta_{9} \ln \left(\text { Solver Expertise }_{j}+1\right) \\
&+\beta_{10} \ln \left(\text { Solver Population }_{j}\right) \\
&+\beta_{11} \ln \left(\text { Feedback-Seeking Volume }_{j}+1\right) \\
&+\beta_{12} \ln \left(\text { Description Length }_{j}+1\right)+\omega_{j} .
\end{aligned}
$$

We run three-stage least square (3SLS) regression analysis and deal with both the simultaneity and the inefficiency issues by jointly estimating Equations (13) and (15). We report the 3SLS estimation results in Table 3. A Durbin-Wu-Hausman test on the full model in column (3-4) indicates that endogeneity is indeed a serious concern in our dataset $\left(\chi^{2}=53.8\right.$ and $\left.p \leq 0.01\right)$.

Column (3-1) presents the results for a 3SLS regression model in which only Feedback Volume and IVs are included. Our results show a positive and statistically significant coefficient of Feedback Volume, thus supporting H1 $\left(\beta_{1}=0.209, S E=0.007, p<0.001\right)$. Particularly, for a $1 \%$ increase in feedback volume, the number of logo designs submitted by each solver increases by $0.21 \%$. With an average solver population of 54.51 , this amounts to $11.45 \%$ increase in the total number of solutions in the contest. Thus, we find strong evidence for the informative role of feedback in mitigating the information asymmetry inherent in ideation contests, thereby inducing solvers to invest more effort in the contest. Additionally, the coefficients of both IVs are statistically significant at the 0.001 level, their signs are as expected, and their values are consistent across other model specifications. These results indicate that feedbackseeking behavior encourages the seeker to provide more feedback comments, while a detailed task description reduces the need for the seeker to offer feedback comments.
Column (3-2) includes CSC in the solver effort model, allowing us to examine the direct effect of CSC on solvers' effort, as proposed in H2. H2(a) suggests that an increase in contest reward can directly motivate higher effort exerted by solvers. As predicted in H2(a), the coefficient of $\ln$ (Contest Reward) is positive and significant at the 0.001 level $\left(\beta_{2}=0.025\right.$, $S E=0.001, p<0.001)$. This finding is consistent with that of the field experiment by Liu et al. (2014), who manipulated the contest reward at two levels, 100 $\mathrm{CNY}$ and $300 \mathrm{CNY}$, and found that solvers submitted more solutions in the high (vs. low) reward condition. The magnitude of the effect in our study is also comparable to theirs. As expected in $\mathrm{H} 2(\mathrm{~b})$, our results suggest a positive effect of contest duration on the number of solutions $\left(\beta_{3}=0.028, S E=0.001\right.$, $p<0.001)$. Our results also indicate a strong correlation between solver effort and expertise, thus supporting H2(c) $\left(\beta_{4}=0.019, S E=0.001, p<0.001\right)$. The coefficient of Solver Population is negative and statistically significant $\left(\beta_{5}=-0.018, S E=0.001, p<0.001\right)$, thus lending support to $\mathrm{H} 2(\mathrm{~d})$. This finding is consistent with that of a similar analysis by Boudreau et al. (2011), who show that solvers' average performance on Topcoder is lowered when the number of solvers increases. In summary, $\mathrm{H} 2$ is supported, confirming the direct effect of CSC on solvers' effort level.

H3(a-d) present hypotheses about how the seeker strategically chooses feedback volume, depending on CSC. To examine $\mathrm{H} 3$, we include CSC in the seeker feedback model and report the results in column (3-3). We find that contest reward is positively associated with feedback volume and is consistent with our predictions in H3(a) $\left(\beta_{6}=0.145, S E=0.008\right.$, $p<0.001)$. The coefficient of contest duration is positive and significantly different from zero, thus confirming $\mathrm{H} 3(\mathrm{~b})\left(\beta_{7}=0.134, S E=0.008, p<0.001\right)$. H3 (c) states that solver expertise has a positive effect on feedback volume, which is supported by our empirical results $\left(\beta_{8}=0.085, S E=0.007, p<0.001\right)$. In addition, we find negative and statistically significant coefficients for solver population, supporting $\mathrm{H} 3(\mathrm{~d})$ $\left(\beta_{9}=-0.089, S E=0.008, p<0.001\right)$. Hence, $\mathrm{H} 3$ is also strongly supported. $\mathrm{H} 3$ also implies the indirect effect that CSC elements have on solvers' effort level through feedback volume. H1-3 collectively suggests that feedback volume plays a partial mediating role in the relationship between CSC and solvers' effort level.

In column (3-4), we present the full model by including CSC on both sides. We find that most coefficients are still statistically significant at the 0.001 level, and the signs of the coefficients are consistent with our hypotheses, except that $\mathrm{H} 2(\mathrm{a}), \mathrm{H} 2(\mathrm{~d})$, and $\mathrm{H} 3(\mathrm{c})$ are left unsupported $\left(\beta_{2}, \beta_{5}\right.$, and $\beta_{8}$ are all insignificant at the 0.05 level). This could be due to variable definitions and operationalization, which we 
Table 3 Three-Stage Least Square Regression Results (Using Number of Solutions to Measure Solver Effort and Average Income to Measure Solver Expertise)

\begin{tabular}{|c|c|c|c|c|c|}
\hline & $3-1$ & $3-2$ & $3-3$ & $3-4$ & Hypothesized sign \\
\hline Solver effort model & \multicolumn{5}{|c|}{ Dependent variable: In(Number of Solutions +1 ) } \\
\hline In(Feedback Volume+1) & $\begin{array}{c}0.209^{* * *} \\
(0.007)\end{array}$ & $\begin{array}{c}0.208^{* * *} \\
(0.007)\end{array}$ & $\begin{array}{c}0.209^{* * *} \\
(0.008)\end{array}$ & $\begin{array}{c}0.208^{* * *} \\
(0.007)\end{array}$ & + \\
\hline In(Contest Reward) & & $\begin{array}{c}0.025^{\star * *} \\
(0.001)\end{array}$ & & $\begin{array}{l}-0.005 \\
(0.003)\end{array}$ & + \\
\hline In(Contest Duration) & & $\begin{array}{c}0.028^{* * *} \\
(0.001)\end{array}$ & & $\begin{array}{c}0.011^{\star *} \\
(0.004)\end{array}$ & + \\
\hline In(Average Income+1) & & $\begin{array}{c}0.019^{\star * *} \\
(0.001)\end{array}$ & & $\begin{array}{c}0.016^{\star \star \star} \\
(0.004)\end{array}$ & + \\
\hline In(Solver Population) & & $\begin{array}{c}-0.018^{\star \star \star *} \\
(0.002)\end{array}$ & & $\begin{array}{l}-0.006 \\
(0.004)\end{array}$ & - \\
\hline Seeker feedback model & \multicolumn{5}{|c|}{ Dependent variable: In(Feedback Volume+1) } \\
\hline In(Contest Reward) & & & $\begin{array}{c}0.145^{\star \star *} \\
(0.008)\end{array}$ & $\begin{array}{c}0.167^{* * *} \\
(0.017)\end{array}$ & + \\
\hline In(Contest Duration) & & & $\begin{array}{c}0.134^{* * *} \\
(0.008)\end{array}$ & $\begin{array}{c}0.088^{* \star *} \\
(0.018)\end{array}$ & + \\
\hline In(Average Income+1) & & & $\begin{array}{c}0.085^{\star * *} \\
(0.007)\end{array}$ & $\begin{array}{c}0.015 \\
(0.017)\end{array}$ & + \\
\hline In(Solver Population) & & & $\begin{array}{c}-0.089^{* * *} \\
(0.008)\end{array}$ & $\begin{array}{c}-0.064^{* * *} \\
(0.019)\end{array}$ & - \\
\hline In(Feedback-Seeking Volume+1) & $\begin{array}{c}0.269^{\star \star \star} \\
(0.008)\end{array}$ & $\begin{array}{c}0.254^{\star \star *} \\
(0.008)\end{array}$ & $\begin{array}{c}0.248^{* * *} \\
(0.008)\end{array}$ & $\begin{array}{c}0.249^{* * *} \\
(0.008)\end{array}$ & + \\
\hline In(Description Length+1) & $\begin{array}{c}-0.073^{* * *} \\
(0.010)\end{array}$ & $\begin{array}{c}-0.184^{* * *} \\
(0.010)\end{array}$ & $\begin{array}{c}-0.213^{\text {*** }} \\
(0.011)\end{array}$ & $\begin{array}{c}-0.213^{* * *} \\
(0.011)\end{array}$ & - \\
\hline Number of observations & 9771 & 9771 & 9771 & 9771 & \\
\hline System-weighted $R^{2}$ & $22.70 \%$ & $24.17 \%$ & $24.05 \%$ & $24.27 \%$ & \\
\hline
\end{tabular}

Note: Standard errors are reported in parentheses; ${ }^{* *} p<0.01 ;{ }^{* * *} p<0.001$.

investigate further in section 5.1. Another potential reason is data aggregation (to the contest level), which adds more noise to the data. Based on the full model estimates in column (3-4), we are able to compute the total effect of CSC on solvers' effort level by combining both the direct and indirect effects. When the seeker increases the reward by $1 \%$, the number of solutions submitted by each solver increases by $0.03 \%$ $\left(=\beta_{2} \times 1 \%+\beta_{1} \times \beta_{7} \times 1 \%\right)$. Similarly, when the duration of the contest is extended by $1 \%$, the number of solutions submitted by each solver increases by $0.03 \%\left(=\beta_{3} \times 1 \%+\beta_{1} \times \beta_{8} \times 1 \%\right)$. When each solver's average income increases by $1 \%$, the number of solutions submitted by each solver increases by $0.02 \%$ $\left(=\beta_{4} \times 1 \%+\beta_{1} \times \beta_{9} \times 1 \%\right)$. When the solver population increases by $1 \%$, the number of solutions submitted by each solver decreases by $0.02 \%$ $\left(=\beta_{5} \times 1 \%+\beta_{1} \times \beta_{10} \times 1 \%\right)$. In light of the large number of participants in each contest (the average being 54.51), these effects are statistically significant as well as economically meaningful.

As a comparison, we also run the OLS, SUR, and 2SLS regression and present the estimation results in Table 4. Column (4-1) reports the OLS results. As expected, OLS estimates of the seeker feedback model are overall consistent with $\mathrm{H} 3$ because they are unbiased. Nonetheless, the solver effort model suffers from the simultaneity issue, leading to biased estimates. Particularly, contrary to the predictions of our theoretical model and the prior literature, we would erroneously conclude that solver effort decreases with contest reward but increases with solver population. This highlights the value of endogenizing seeker's feedback decision and taking account of the endogeneity issue that arises from endogenous feedback volume. Then we run the SUR regression with coefficients reported in column (4-2). Compared with the OLS estimates in column (4-1), the efficiency of SUR coefficients has improved, but they are still biased and may have the wrong sign. As shown in column (4-3), 2SLS corrects the endogeneity bias but does not resolve the inefficiency issue.

\section{Robustness and Model Extensions}

In this section, we relax some assumptions of the theoretical model and perform various empirical checks to establish the robustness of our results.

\subsection{Alternative Variable Definitions and Operationalization}

We first investigate whether our empirical results are sensitive to variable definitions and operationalization. Two variables that are measured by surrogates and 
Table 4 OLS, SUR, and 2SLS Regression Results (Using Number of Solutions to Measure Solver Effort and Average Income to Measure Solver Expertise)

\begin{tabular}{|c|c|c|c|c|}
\hline & $4-1$ & $4-2$ & $4-3$ & \\
\hline & OLS & SUR & 2SLS & sign \\
\hline $\begin{array}{l}\text { Solver effort } \\
\text { model }\end{array}$ & \multicolumn{4}{|c|}{ Dependent variable: In(Number of Solutions +1$)$} \\
\hline In(Feedback Volume+1) & $\begin{array}{c}0.035^{\star * *} \\
(0.001)\end{array}$ & $\begin{array}{c}0.048^{* * *} \\
(0.001)\end{array}$ & $\begin{array}{c}0.208^{* * *} \\
(0.007)\end{array}$ & + \\
\hline In(Contest Reward) & $\begin{array}{l}-0.020^{* * *} \\
(0.002)\end{array}$ & $\begin{array}{l}-0.022^{* * *} \\
(0.002)\end{array}$ & $\begin{array}{l}-0.005 \\
(0.004)\end{array}$ & + \\
\hline In(Contest Duration) & $\begin{array}{c}0.013^{* * *} \\
(0.002)\end{array}$ & $\begin{array}{c}0.011^{* * *} \\
(0.002)\end{array}$ & $\begin{array}{l}0.011^{\star *} \\
(0.004)\end{array}$ & + \\
\hline In(Average Income +1$)$ & $\begin{array}{c}0.018^{\star * *} \\
(0.002)\end{array}$ & $\begin{array}{c}0.018^{* * *} \\
(0.002)\end{array}$ & $\begin{array}{c}0.016^{\star \star *} \\
(0.004)\end{array}$ & + \\
\hline In(Solver Population) & $\begin{array}{l}0.018^{* * *} \\
(0.002)\end{array}$ & $\begin{array}{l}0.017^{\star * *} \\
(0.002)\end{array}$ & $\begin{array}{l}-0.006 \\
(0.004)\end{array}$ & - \\
\hline Adjusted $R^{2}$ & $14.40 \%$ & - & $9.33 \%$ & \\
\hline Seeker feedback model & \multicolumn{4}{|c|}{ Dependent variable: In(Feedback Volume+1) } \\
\hline In(Contest Reward) & $\begin{array}{c}0.107^{\star * \star} \\
(0.018)\end{array}$ & $\begin{array}{c}0.206^{* * *} \\
(0.018)\end{array}$ & $\begin{array}{c}0.212^{\star \star \star} \\
(0.018)\end{array}$ & + \\
\hline In(Contest Duration) & $\begin{array}{c}0.118^{\star \star \star} \\
(0.019)\end{array}$ & $\begin{array}{c}0.081^{\star \star *} \\
(0.018)\end{array}$ & $\begin{array}{c}0.089^{* \star *} \\
(0.018)\end{array}$ & + \\
\hline In(Average Income +1$)$ & $\begin{array}{l}0.028^{\ulcorner} \\
(0.018)\end{array}$ & $\begin{array}{c}0.011 \\
(0.017)\end{array}$ & $\begin{array}{c}0.013 \\
(0.017)\end{array}$ & + \\
\hline In(Solver Population) & $\begin{array}{l}-0.057^{* *} \\
(0.020)\end{array}$ & $\begin{array}{c}-0.091^{* * *} \\
(0.019)\end{array}$ & $\begin{array}{l}-0.058^{* *} \\
(0.019)\end{array}$ & - \\
\hline In(Feedback-Seeking Volume+1) & - & - & $\begin{array}{c}0.227^{\star \star *} \\
(0.009)\end{array}$ & + \\
\hline In(Description Length+1) & - & - & $\begin{array}{c}-0.361^{* * *} \\
(0.022)\end{array}$ & - \\
\hline Adjusted $R^{2}$ & $2.61 \%$ & - & $11.68 \%$ & \\
\hline System-weighted $R^{2}$ & - & $20.58 \%$ & - & \\
\hline Number of observations & 9771 & 9771 & 9771 & \\
\hline
\end{tabular}

Note: Standard errors are reported in parentheses; $\left\ulcorner p<0.1 ;{ }^{\star \star} p<0.01 ;{ }^{* \star *} p<0.001\right.$.

thus may suffer from this issue are solver effort and expertise. As such, we employ an alternative measure of solvers' effort, Elapsed Time, defined as the amount of time solvers spent on the contest. It is calculated as the difference between the registration date and the last submission date in the unit of days. Then, Elapsed Time is averaged across all solvers in the contest to obtain the contest-level measure. This method is consistent with the project management literature in which effort is usually measured as the amount of time required to complete a task (e.g., workdays) (Kerzner 1995). ${ }^{13}$ Additionally, Elapsed Time is unlikely to be correlated with Feedback-Seeking Volume (the IV for Feedback Volume). This is because the time it takes solvers to write text messages to request feedback is negligible compared with the time they spent in the contest. Similarly, we do not see an obvious link between Elapsed Time and Description Length.

Two alternative measures of solver expertise are Tenure and Experience. Tenure is defined as the number of months elapsed since the solver participated in his first contest, while Experience is defined as the total number of contests in which a solver has participated before joining the contest. It is easy to see that both Tenure and Experience contribute to the accumulation of knowledge, skills, and experience. Both measures are averaged, respectively, over all solvers in the contest to obtain contest-level solver expertise.

We replicate our analysis using these alternative measures of solver effort and solver expertise. Estimation results are reported in Table 5. Columns (5-1) and (5-2) show the robustness of our main specification using Tenure and Experience as alternative measures of solver expertise. We find that, in addition to all the empirically supported hypotheses in section 4.4 , the effect of solver expertise on feedback volume becomes significant at the 0.01 level in both models, therefore supporting H3(c). Columns (5-3), (5-4), and (5-5) show that all three hypotheses (H1-3) are strongly supported when Elapsed Time is used to measure solver effort and Average Income, Tenure, and Experience are used to measure solver expertise, respectively. These estimation results suggest that our empirical results are robust, offering strong support to our theory. 
Table 5 Three-Stage Least Square Regression Results (Using Alternative Measures of Solver Effort and Solver Expertise)

\begin{tabular}{|c|c|c|c|c|c|c|}
\hline & $5-1$ & $5-2$ & $5-3$ & $5-4$ & $5-5$ & \\
\hline Measure of solver effort & Number of Solutions & Number of Solutions & Elapsed Time & Elapsed Time & Elapsed Time & \\
\hline Measure of solver expertise & Tenure & Experience & Average Income & Tenure & Experience & Hypothesized sign \\
\hline Solver effort model & \multicolumn{6}{|c|}{ Dependent variable: In(Effort+1) } \\
\hline In(Feedback Volume+1) & $\begin{array}{c}0.209^{* * *} \\
(0.007)\end{array}$ & $\begin{array}{c}0.209^{* * *} \\
(0.007)\end{array}$ & $\begin{array}{c}0.172^{* * *} \\
(0.010)\end{array}$ & $\begin{array}{c}0.176^{* * *} \\
(0.010)\end{array}$ & $\begin{array}{c}0.179^{* * *} \\
(0.010)\end{array}$ & + \\
\hline In(Contest Reward) & $\begin{array}{l}-0.001 \\
(0.003)\end{array}$ & $\begin{array}{c}0.001 \\
(0.003)\end{array}$ & $\begin{array}{l}0.008^{\ulcorner} \\
(0.005)\end{array}$ & $\begin{array}{c}0.027^{\star \star \star} \\
(0.005)\end{array}$ & $\begin{array}{c}0.042^{\star \star \star} \\
(0.005)\end{array}$ & + \\
\hline In(Contest Duration) & $\begin{array}{l}0.011^{* *} \\
(0.004)\end{array}$ & $\begin{array}{l}0.010^{* *} \\
(0.004)\end{array}$ & $\begin{array}{c}0.804^{\star \star \star *} \\
(0.005)\end{array}$ & $\begin{array}{c}0.807^{\star * *} \\
(0.005)\end{array}$ & $\begin{array}{c}0.801^{* * *} \\
(0.006)\end{array}$ & + \\
\hline In(Solver Expertise+ 1) & $\begin{array}{c}0.022^{* \star *} \\
(0.006)\end{array}$ & $\begin{array}{c}0.000 \\
(0.002)\end{array}$ & $\begin{array}{c}0.092^{* * *} \\
(0.005)\end{array}$ & $\begin{array}{c}0.155^{\star \star *} \\
(0.008)\end{array}$ & $\begin{array}{c}0.011^{* * *} \\
(0.03)\end{array}$ & + \\
\hline In(Solver Population) & $\begin{array}{l}-0.004 \\
(0.004)\end{array}$ & $\begin{array}{l}-0.005 \\
(0.004)\end{array}$ & $\begin{array}{c}-0.023^{* \star *} \\
(0.006)\end{array}$ & $\begin{array}{c}-0.014^{\star} \\
(0.006)\end{array}$ & $\begin{array}{c}-0.019^{* * *} \\
(0.006)\end{array}$ & - \\
\hline Seeker feedback model & \multicolumn{6}{|c|}{ Dependent variable: In(Feedback Volume+1) } \\
\hline In(Contest Reward) & $\begin{array}{c}0.165^{\star * *} \\
(0.016)\end{array}$ & $\begin{array}{l}0.171^{* * *} \\
(0.016)\end{array}$ & $\begin{array}{c}0.187^{\star \star \star} \\
(0.018)\end{array}$ & $\begin{array}{c}0.183^{* * *} \\
(0.017)\end{array}$ & $\begin{array}{c}0.191^{\star * *} \\
(0.017)\end{array}$ & + \\
\hline In(Contest Duration) & $\begin{array}{c}0.090^{* \star *} \\
(0.018)\end{array}$ & $\begin{array}{c}0.091^{* * *} \\
(0.018)\end{array}$ & $\begin{array}{c}0.088^{\star \star *} \\
(0.018)\end{array}$ & $\begin{array}{c}0.090^{* * *} \\
(0.018)\end{array}$ & $\begin{array}{c}0.091^{* * *} \\
(0.018)\end{array}$ & + \\
\hline In(Solver Expertise+ 1) & $\begin{array}{l}0.067^{\star \star} \\
(0.026)\end{array}$ & $\begin{array}{c}0.048^{\star \star *} \\
(0.010)\end{array}$ & $\begin{array}{c}0.014 \\
(0.017)\end{array}$ & $\begin{array}{l}0.065^{\star} \\
(0.026)\end{array}$ & $\begin{array}{c}0.046^{\star \star \star *} \\
(0.010)\end{array}$ & + \\
\hline In(Solver Population) & $\begin{array}{c}-0.062^{* * *} \\
(0.019)\end{array}$ & $\begin{array}{c}-0.067^{\star * \star} \\
(0.019)\end{array}$ & $\begin{array}{c}-0.062^{* * *} \\
(0.019)\end{array}$ & $\begin{array}{c}-0.060^{* *} \\
(0.019)\end{array}$ & $\begin{array}{c}-0.064^{* * *} \\
(0.019)\end{array}$ & - \\
\hline In(Feedback-Seeking Volume+1) & $\begin{array}{c}0.249^{* * *} \\
(0.008)\end{array}$ & $\begin{array}{c}0.253^{* * *} \\
(0.008)\end{array}$ & $\begin{array}{c}0.239 * * * \\
(0.009)\end{array}$ & $\begin{array}{c}0.241^{* * *} \\
(0.009)\end{array}$ & $\begin{array}{c}0.243^{* * *} \\
(0.009)\end{array}$ & + \\
\hline In(Description Length+1) & $\begin{array}{c}-0.212^{* * *} \\
(0.011)\end{array}$ & $\begin{array}{c}-0.212^{* * *} \\
(0.011)\end{array}$ & $\begin{array}{c}-0.277^{\star * *} \\
(0.017)\end{array}$ & $\begin{array}{c}-0.270^{* \star *} \\
(0.017)\end{array}$ & $\begin{array}{c}-0.276^{* * *} \\
(0.017)\end{array}$ & - \\
\hline Number of observations & 9771 & 9771 & 9771 & 9771 & 9771 & \\
\hline System-weighted $R^{2}$ & $24.08 \%$ & $23.97 \%$ & $70.76 \%$ & $70.78 \%$ & $69.46 \%$ & \\
\hline
\end{tabular}

Note: Standard errors are reported in parentheses; $\left\ulcorner p<0.1 ;{ }^{\star} p<0.05 ;{ }^{* \star} p<0.01 ;{ }^{* \star} p<0.001\right.$.

Recall that, before taking the logarithm, we add one to variables that may take the value of zero: $\ln \left(\right.$ Variable $\left._{j}+1\right)$. This includes variables such as Number of Solutions, Average Income, Feedback Volume, Feedback-Seeking Volume, and Description Length. To examine whether this approach drives our results, we employ the following variable operationalization:

$$
\ln \left(\text { Variable }_{j}+X\right)
$$

where $X>0$. We re-estimate the main model (Number of Solutions and Average Income as measures of solver effort and expertise, respectively) by varying the value of $X: X=0.01, X=0.1, X=0.5$, and $X=2$. Table A2 in Online Appendix A summarizes the results. The case of $X=1$ is shown in column (A2-4) for ease of comparison.

Overall, variable operationalization may influence the significance and effect size of the model coefficients but not their signs. A closer examination reveals that $\beta_{4}$ is negative and statistically significant at the 0.05 level for all values of $X$ except for $X=1$, lending additional support to $\mathrm{H} 2(\mathrm{~d})$ that larger solver population decreases solver effort; $\beta_{9}$ becomes statistically significant as $X$ decreases, providing some support to $\mathrm{H} 3(\mathrm{c})$ that higher solver expertise leads to higher feedback volume; however, $\beta_{2}$ is still insignificant under various values of $X$. All other significant estimates in the main model remain unchanged.

To summarize, all hypotheses are supported by the main model (see Table 3) except for H2(a), H2(d), and H3(c). Further exploration uncovers that the estimates of these three effects appear to be sensitive to variable definitions and operationalization, while all other results remain extremely robust. In particular, when Elapsed Time is used as the measure of solver effort, all hypothesized effects-including $\mathrm{H} 2$ (a), H2(d) and $\mathrm{H} 3$ (c) - are supported empirically (see Table 5). This may suggest that solvers tend to devote significantly more time working on the logo design in response to higher contest reward and smaller solver population without increasing submissions. Furthermore, when solver effort is measured by Number of Solutions, the effect of solver expertise, measured by Tenure or Experience, on feedback volume is significant, lending support to H3(c) (see Table 5). The effect of Solver Population on Solver Effort appears to be sensitive to $X$ in $\ln \left(\right.$ Variable $\left._{j}+X\right)$, with $\mathrm{H} 2(\mathrm{~d})$ being supported for all values of $X$ except for $X=1$ (see Table A2). Taken 
together, most hypothesized effects are robust to variable definitions and operationalization. Thus, the theoretical predictions are largely supported by our empirical analysis.

\subsection{Heterogeneous Solvers}

Our analytical model assumes that all solvers in the same contest are homogeneous. This assumption greatly simplifies the analysis and is necessary for deriving the closed-form solutions. However, in practice, ideation contests naturally attract contestants from a heterogeneous pool of solvers. As demonstrated in our data, solvers competing in the same contest generally have different levels of expertise. This creates a slight disconnect between the homogeneity assumption and the actual data, even though we take average of solver expertise across all solvers in the contest to obtain the contest-level measure. In this subsection, we test the robustness of our findings by varying the level of solver heterogeneity.

To this end, for each contest we calculate the standard deviation of solver expertise measured by Average Income. Then we divide all contests into two halves by performing a median split based on the standard deviation of solver expertise. In this way, we obtain two subsamples with the "above-median" ("below-median") sample consisting of contests with relatively heterogeneous (homogeneous) solvers. We run 3SLS regression models separately with both subsamples using Average Income as the measure of solver expertise and alternative measures of solver effort. We also follow the same procedure for the other two measures of solver expertise: Tenure and Experience. Estimation results are summarized in Tables A3 and A4 in Online Appendix A. We find that the two subsamples produce qualitatively the same results as the main model. This suggests that, despite the homogeneity assumption in the theoretical model, analysis using subsamples of more (or less) heterogeneous solvers does not affect our main results.

\subsection{Endogenous Solver Entry}

In the main model, we assume that solvers' entry into the contest is exogenous (i.e., solver population $N$ is exogenously determined). Now we relax this assumption by allowing solvers to decide whether to enter the contest. Let $\bar{N}$ ( $\bar{N}$ is sufficiently large) be the total number of solvers who consider whether to participate. The game sequence is as follows: the seeker chooses feedback volume in stage one; solvers simultaneously decide whether to enter the contest in stage two; and solvers expend effort in stage three. We solve the model by backward induction. It is easy to see that the solution of stage three remains the same so we obtain from Equation (10) the equilibrium effort level $e^{*}(N(M), M)$ as a function of feedback volume $M$ and solver population $N(M)$.

Then we go back to stage two and characterize solvers' entry decision. An increase in solver population has two effects on solvers. The expected benefit of entering the contest decreases because a larger solver population reduces the likelihood of winning. This is termed benefit-reduction effect of entry. We also identify a cost-reduction effect when endogenizing solvers' effort level: the cost of entering the contest decreases because a larger solver population lowers solvers' effort and the associated cost. To obtain an interior solution, we assume that $\alpha(T)$ is sufficiently large, that is, $\alpha(T)>\overline{\bar{\alpha}}(T)$ where $\overline{\bar{\alpha}}(T)$ is defined in Online Appendix B. With this assumption, the benefit-reduction effect outweighs the cost-reduction effect. The net effect is such that solvers' utility from entering the contest decreases with solver population. Solvers' equilibrium entry strategy is formally presented in the following proposition.

Proposition 3. There exists a unique mixed-strategy Nash equilibrium in solvers' entry into the contest. The probability that each solver enters the contest is $\frac{N^{*}}{N}$ where $N^{*}$ is the equilibrium solver population and is the unique solution of

$$
\frac{2^{N^{*}+2}}{\left(N^{*}-1\right)^{2}}=\frac{a^{2} V\left(M \tau^{2}+\sigma^{2}\right)^{2}}{\pi \alpha(T) \tau^{4} \sigma^{4}} .
$$

$N^{*}$ increases with feedback volume, contest reward, contest duration, and solver expertise.

Proof. See Online Appendix B.

Since solvers are worse off when more competitors join the contest, it is never optimal for all of them to enter the contest in equilibrium because they will obtain negative utility by doing so. Thus, solvers adopt a mixed strategy where some of them enter the contest while others do not. In equilibrium, all solvers obtain an expected utility of zero, that is, they are indifferent to entering the contest or not. We find that the equilibrium solver population increases with feedback volume, contest reward, contest duration, and solver expertise. This result can be attributed to their impacts on the benefitand cost-reduction effects. An increase in feedback volume, contest reward, and solver expertise magnifies both benefit- and cost-reduction effects. Since the cost-reduction effect increases as a faster pace than the benefit-reduction effect, the equilibrium solver population increases with these variables. Similarly, an increase in contest duration weakens both effects but the cost-reduction effect decreases 
more slowly than the benefit-reduction effect. As a result, the equilibrium solver population increases with contest duration. ${ }^{14}$

\subsection{Pre-Feedback Solver Effort}

The sequence of decisions in the main theoretic model is intuitive as it reflects the relative flexibility in the decision-making process: solvers have a lot of flexibility in exerting more effort after receiving the seeker's feedback comments. Nonetheless, feedback is usually given based on submitted solutions, indicating that solvers must have already invested some effort before any feedback is given. To allow for this possibility, we now consider a three-stage model in which solvers can exert effort both before and after the seeker's feedback decision is made. More specifically, solvers expend pre-feedback effort in stage one, the seeker decides on feedback volume in stage two, and solvers choose the post-feedback effort in stage three. We assume that the seeker offers feedback to solvers at some time when the contest is running. Without loss of generality, let the time at which feedback is provided be $\theta T$ where $\theta \in(0,1)$ and $T$ is the contest druation. With this assumption, the pre-feedback duration is $\theta T$ and the post-feedback duration is $(1-\theta) T$. We also restrict our attention to the symmetric Nash equilibrium in which all solvers share the same pre- and post-feedback efforts. Detailed proof is provided in Online Appendix B.

In this model, solver i's performance depends on both pre- and post-feedback effort:

$$
d_{i}=a\left(e_{i}^{\text {pre }}+e_{i}^{\text {post }}\right)+\mu_{i} .
$$

We solve the model by backward induction, beginning with solving the stage-three decision while taking pre-feedback efforts and feedback volume as given. Solvers' utility maximization problem is written as

$\max _{e_{i}^{\text {post }}} U\left(e_{i}^{\text {post }} \mid e_{i}^{\text {pre }}, M\right)=V \operatorname{Pr}\left(w_{i n}\right)-\alpha((1-\theta) T) e_{i}^{\text {post2 }}$,

where $\operatorname{Pr}\left(\operatorname{win}_{i}\right)$ is solver $i^{\prime}$ s likelihood of winning the contest, given by

$$
\begin{aligned}
\operatorname{Pr}\left(\operatorname{win}_{i}\right) & =\prod_{k \neq i} \operatorname{Pr}\left(d_{i}>d_{k} \mid \mathbf{X}\right) \\
& =\prod_{k \neq i} \Phi\left(\frac{a\left(e_{i}^{\text {post }}+e_{i}^{\text {pre }}-e_{k}^{\text {post }}-e_{k}^{\text {pre }}\right)\left(M \tau^{2}+\sigma^{2}\right)}{2 \tau^{2} \sigma^{2}}\right) .
\end{aligned}
$$

By invoking symmetry $e_{i}^{\text {pre }}=e_{k}^{\text {pre }}$, we obtain the equilibrium post-feedback effort:

$$
e^{\text {post* }}=\frac{a V(N-1)\left(M \tau^{2}+\sigma^{2}\right)}{2^{N+\frac{1}{2}} \sqrt{\pi} \alpha((1-\theta) T) \tau^{2} \sigma^{2}} .
$$

It is apparent that the post-feedback effort level increases with feedback volume, contest reward, contest duration, and solver expertise, and decreases with solver population. Then we go back to stage two and analyze the seeker's decision on feedback volume. The profit of the seeker is the same as in Equation (11). The seeker maximizes her profit by choosing feedback volume $M$. By solving this profit maximization problem, we obtain

$$
M^{*}=\frac{\sigma}{\tau} \sqrt{\frac{\mu_{\text {feedback }}-\mu_{\text {prior }}-\tau^{2} \int_{-\infty}^{\infty} z d \Phi(z)^{N}}{\beta-a e^{p r e}-\frac{a^{2} V(N-1)}{2^{N+\frac{1}{2}} \sqrt{\pi} \alpha((1-\theta) T) \sigma^{2}}}}-\frac{\sigma^{2}}{\tau^{2}} .
$$

It can be shown that feedback volume increases with pre-feedback effort, contest reward, contest duration, solver expertise, and decreases with solver population. Finally, we go back to stage one and evaluate solvers' pre-feedback efforts. We assume that solvers have rational expectations on their postfeedback effort level, with utility

$$
\begin{aligned}
U\left(e_{i}^{\text {pre }}\right) & =V \prod_{k \neq i} \Phi\left(\frac{a\left(e_{i}^{\text {pre }}+e_{i}^{\text {post }}-e_{k}^{\text {pre }}-e_{k}^{\text {post }}\right)\left(M^{*} \tau^{2}+\sigma^{2}\right)}{2 \tau^{2} \sigma^{2}}\right) \\
& -\alpha(\theta T) e_{i}^{\text {pre } 2} .
\end{aligned}
$$

Thus, we have $e_{i}^{\text {post }}=e_{k}^{\text {post }}=e^{\text {post }}$. All solvers simultaneously choose their pre-feedback effort level by maximizing their utility function, which can be rewritten as follows:

$$
\begin{aligned}
\max _{e_{i}^{p r e}} U\left(e_{i}^{\text {pre }}\right) & =V \prod_{k \neq i} \Phi\left(\frac{a\left(e_{i}^{\text {pre }}-e_{k}^{\text {pre }}\right)\left(M^{*} \tau^{2}+\sigma^{2}\right)}{2 \tau^{2} \sigma^{2}}\right) \\
& -\alpha(\theta T) e_{i}^{\text {pre2 }} .
\end{aligned}
$$

We find that there exists an optimal pre-feedback effort $e_{i}^{\text {pre* }}$ that satisfies the following equation:

$$
\begin{aligned}
& e^{\text {pre*2 }}\left(\beta-\frac{a^{2} V(N-1)}{2^{N+\frac{1}{2}} \sqrt{\pi} \alpha((1-\theta) T) \sigma^{2}}-a e^{\text {pre* }}\right) \\
& -\frac{a^{2} V^{2}(N-1)^{2}\left(\mu_{\text {feedback }}-\mu_{\text {prior }}-\tau^{2} \int_{-\infty}^{\infty} z d \Phi(z)^{N}\right)}{2^{N+\frac{1}{2}} \pi \tau^{2} \sigma^{2} \alpha^{2}(\theta T)}=0 .
\end{aligned}
$$

Furthermore, $e^{\text {pre* }}$ increases with contest reward, contest duration, and solver expertise, and decreases 
with solver population. (Detailed proof is provided in Online Appendix B.) From the above analysis, we conclude that all three sets of hypotheses in section 3.3 still hold in this three-stage model.

\section{Implications and Conclusions}

Advances in information technology have fundamentally transformed how companies engage in ideation processes. In today's business world, many companies rely on ideation contests to complete tasks that were previously performed by employees or contractors. Harnessing the power of the Internet, ideation contests allow companies to tap into "the wisdom of the crowd" by soliciting a large number of solutions at minimal costs. Although ideation contests are widely adopted by businesses, little is known about how feedback is used and how it affects solvers' effort level.

In this study, we build a theoretical model of ideation contest that incorporates the feedback mechanism through which seekers reveal their private taste to solvers. Ideation contests are characterized by information asymmetry - the seekers' private information about their taste is imperfectly known to solvers. Information asymmetry leads to market inefficiency and solvers' suboptimal behavior. We find that the solvers' effort level increases as seekers provide more feedback because it can help reduce solvers' uncertainty about the seekers' taste. We also find a direct effect of contest and solver characteristics (CSC) on solvers' effort level. The solvers' equilibrium effort level increases with contest reward, contest duration, and solver expertise but decreases with solver population. By endogenizing the seeker's feedback volume, we find that feedback partially mediates the relationship between CSC and the solvers' effort level. Thus, CSC elements also have an indirect effect on solvers' effort level through the seeker's feedback.

Theoretical hypotheses are tested using data from 9771 contests hosted by Zhubajie.com. The empirical analysis provides strong support for our theory. Various robustness checks and model extensions are conducted to corroborate the robustness of our findings. Our empirical results are invariant when employing alternative variable definitions and operationalization, or using a relatively homogeneous or heterogeneous subsample. In addition, we extend the base model to endogenize solvers' entry decision and expand to a three-stage game that allows solvers to expend effort before feedback is given. These model extensions lend further support to our main analytical results and propositions.

\subsection{Theoretical and Managerial Implications}

We investigate the role of seeker's feedback in ideation contests using a combination of theoretical and empirical analyses. The informative role of feedback suggests that seekers should be strongly encouraged to provide comments on solvers' submissions. This can be achieved by educating seekers regarding the importance of giving feedback, which is consistent with the practice of several ideation contest platforms (e.g., 99Designs and DesignContest), although some other platforms remain silent and may therefore suffer from an undersupply of feedback. In addition, ideation contest platforms can focus on increasing the benefits and reducing the costs of feedback provision. This can be done by awarding seekers who actively leave feedback with extra bonuses and by investing in easy-to-use and efficient feedback systems. Platforms should also encourage solvers to engage in feedbackseeking behaviors. For example, Zhubajie recommends to solvers, "Frequent communication with the employer (seeker) during the work process will help you to deliver better results." ${ }^{\prime 15}$

One challenge that seekers encounter in ideation contests involves the design and management of CSC. Using a dataset with substantial variation in contest design, we examine four CSC elements concurrently in this study. Firstly, we show that higher contest rewards incentivize greater solver effort. Although Liu et al. (2014) find a similar result in a field experiment on Taskcn.com, they only consider two reward levels: $100 \mathrm{CNY}$ and $300 \mathrm{CNY}$. Our result is more general and suggests that firms seeking ideation solutions via contests should carefully select appropriate reward amounts to achieve optimal outcomes. This monetary aspect also has practical implications for platforms as their primary revenue source comprises the transaction fee charged at a certain percentage of the contest rewards. Secondly, our study is the first to show that contest duration can positively affect solvers' effort. Therefore, seekers must balance their requirements for high-quality solutions with their desire for rapid delivery time. Thirdly, we demonstrate that solvers with greater expertise tend to spend more time working on the task and are more likely to deliver solutions in larger quantity and with higher quality. Therefore, it is important for seekers to keep track of solver profiles and to make sure that capable and experienced solvers can participate. To do so, seekers can strategically invite the most promising solvers to join the contests (Mo et al. 2018). For example, 99Designs and DesignCrowd allow seekers to browse through the pool of solvers and send invitations directly to individual solvers. Moreover, it is in the platforms' best interest to build a large pool of highly-skilled solvers and encourage their active participation using various incentives. This strategy is critical to seekers' satisfaction and the healthy growth of the platform. Lastly, our theory predicts that increased competition among solvers tends to 
dampen their effort level, a finding that is consistent with previous literature (e.g., Boudreau et al. 2011, Terwiesch and $\mathrm{Xu}$ 2008). However, as Terwiesch and $\mathrm{Xu}$ (2008) point out, a larger solver population is not necessarily bad for seekers, especially when they are looking for a single best solution.

Another important finding of this study is the mediating role of feedback in the relationship between CSC and the solvers' effort level, which has profound theoretical and managerial implications. Previous literature usually takes the seekers' feedback strategy as exogenous, while our paper takes the first step toward proposing and validating that seekers are strategic in determining whether to provide feedback and the feedback volume. Therefore, when assessing the total effect of CSC on solvers' effort level, firms should consider both the direct effect and the indirect effect through feedback volume. Otherwise, the design of CSC could be suboptimal. We also recognize that the solver-side effort model suffers from the endogeneity bias if the seekers' strategic decisions regarding feedback volume are not taken into account. Ideation contests designed without this consideration could be counterproductive. To the best of our knowledge, this study is the first to investigate feedback as a critical mediator in the CSC-effort relationship.

\subsection{Limitations and Directions for Future \\ Research}

Our research is not exempt from limitations, which suggest future research directions. Our analytical model features an ideation contest with symmetric solvers. Although this assumption greatly simplifies our analysis, it may not reflect the reality of the ideation contest markets. More specifically, there exists substantial heterogeneity in solvers' expertise and capability of extracting information from feedback. Consequently, solvers are not symmetric as in the base model. It would also be interesting for future studies to further investigate whether solvers with varying levels of expertise would respond differently to seekers' feedback. If so, the effectiveness of feedback in motivating solver effort might be moderated by solver expertise. Another interesting topic is to study how feedback affects the distribution of solver expertise in a contest, when solver entry is endogenous. Additionally, solvers with various types of expertise might respond differently to CSC. For example, it would be interesting to investigate whether rewards would be more useful in driving solvers with high or low expertise to expend greater efforts.

We have assumed that solvers' match values with the seeker follow an identical distribution. In reality, some solvers might have previous experience of working for certain seekers and would be more knowledgeable about these seekers' private taste than others. These more experienced solvers might start with a different distribution of match values, and may also interpret feedback differently than those with little prior knowledge. However, we note that numerical analysis in this case is necessary as there is no closed-form solution in Bayesian learning framework. Future research should examine how solvers' prior information would affect their strategic behavior in ideation contests.

The limitations of our dataset should also be noted. Although we use instrumental variables such as Feedback-Seeking Volume to correct potential endogeneity bias, we lack instruments or control variables to fully establish causality, although our empirical results are consistent with theoretical predictions. Future research can investigate a richer context with more options of instrumental variables and control variables.

Finally, since it is important to demonstrate the generalizability of these results, corroborating our findings in subsequent research would be quite useful, especially across platforms and across service categories. It is possible that both the strength of the informative and mediating roles of feedback are contingent on the nature of the platform and the service category, as the level of information asymmetry is essential in driving our theoretical predictions.

\section{Acknowledgments}

We are immensely grateful to the Department Editor, the Senior Editor, and the three anonymous reviewers for their constructive comments that greatly helped us improve the paper. We thank the seminar participants at Virginia Tech, our audience at the 37th Annual ISMS Marketing Science conference, and Dr. Sam Min at California State University, Long Beach for their insightful feedback on earlier drafts of the manuscript.

\begin{abstract}
Notes
${ }^{1}$ Indeed, ideation contest platforms such as 99Designs and DesignContest have recognized the importance of feedback in ideation contests and actively encourage it. For instance, 99Designs recommends to seekers, "Leaving a comment is the most important and productive way of providing feedback, as it allows you to interact with the designers and provide guidance. Tell designers if their design is going in the right direction, and how they can improve their submission." DesignContest emphasizes, "Evaluating entries and communicating with designers help you to get exactly the design you're looking for."

${ }^{2}$ It is worth noting that our work is related to but different from the freelance literature. Freelancers compete in marketplaces to take on jobs such as web development, programming, writing, and translation. This involves an
\end{abstract}


auction-like procedure, ending with a contract signed by the buyer and the winning freelancer. Selection of the winner is based on participating freelancers' initial bids and other observable characteristics such as reputation. The selected freelancer starts his work after he wins the contract. This implicates that the buyer's main challenge lies in the information asymmetry caused by a potential "lemon" problem (see, e.g., Gandini 2016, Yoganarasimhan 2013, 2015). Since the buyer only receives the work (solution) only from the winning freelancer, she does not benefit from the "wisdom of the crowd," which is in stark contrast to the ideation contests we study.

${ }^{3}$ Ideation contest bears considerable similarity with sales contest that has received much attention in the marketing literature (see Chen and Lim 2013, 2017, Chen et al. 2011, Kalra and Shi 2001, Lim 2010, Lim et al. 2009, to name a few). However, they are different in two important ways: (i) The seeker in an ideation contest usually seeks and pays for the favorite solution among all submissions, whereas the purpose of sales contests is to increase the overall output of all contestants; (ii) In ideation contests solvers act on a completely voluntary basis, whereas salespersons are often obliged to participate in sales contests. Thus, characteristics of these contests are fundamentally different, as is evident in our model in section 3.

${ }^{4}$ There is a large body of research on performance feedback in contests and tournaments in the economics and business literature (see Aoyagi 2010, Ederer 2010, Moldovanu and Sela 2001, to name a few), which unanimously examines feedback that contains information about contestants' performance relative to others. Such performance feedback is treated as a signal of contestants' hidden effort. In contrast, we study feedback comments that reveal the seeker's private taste. It is also noteworthy that in ideation contests, solvers' effort level (or its proxy) is publicly known. Thus, prior research findings on performance feedback cannot be generalized to the context of ideation contests.

${ }^{5}$ In section 5.4, we extend the model by allowing solvers to exert effort before feedback is given.

${ }^{6}$ If solvers' performance is assumed to take an additive form of solver expertise and effort, that is, $d_{i}=a+e_{i}+\mu_{i}$, solver expertise will be cancelled out when comparing two different solvers' performance and hence does not affect the equilibrium effort level.

${ }^{7}$ In this model, solvers are assumed to be homogenous. In Online Appendix C, we extend the theoretical model to allow for solver heterogeneity. Simulation analysis suggests that the main findings remain unchanged and, moreover, solvers with higher expertise expend greater efforts than those with lower expertise. We also empirically investigate the implications of solver heterogeneity in section 5.2.

${ }^{8}$ Source: http://www.witmart.com/ and http://techcrunc h.com/2012/12/08/asias-secret-crowdsourcing-boom/ (accessed date July 1, 2019).

${ }^{9}$ Our results are almost invariant when we include contests with reward below $50 \mathrm{CNY}$.

${ }^{10}$ Submission of a revised logo design is counted as another solution.
${ }^{11}$ In practice, the seeker can terminate the contest prior to the initially proposed end date. Thus, the actual contest duration could be different (shorter) than the proposed contest duration. Our empirical results are similar when the proposed contest duration is used.

${ }^{12} \mathrm{In}$ Equation (13) FeedbackVolume $e_{i}$ is correlated with $\varepsilon_{i}$ because FeedbackVolume $i$ is a function of $v_{i}$ as seen in Equation (14), thus leading to the simultaneity issue. Notably, Equation (14) suffers from inefficiency yet has no endogeneity issue.

${ }^{13} \mathrm{~A}$ potential concern for using elapsed time to measure effort is that if solvers intentionally postpone submitting their designs until the last minute, then elapsed time inaccurately reflects the amount of time they actually spend on the contests. However, this is unlikely in our context. In our data, over $80 \%$ of solvers registered on the first day of the contest, and only $5 \%$ of solutions are submitted on the last day of the contest. Moreover, the mean of Elapsed Time is 3.69 days, which is about $34 \%$ of contest duration, indicating that procrastination is an uncommon practice in our context. There are at least two reasons for this. First, Zhubajie allows the seeker to terminate the contest earlier than the proposed ending time without prior notice once a satisfactory logo design has been received. Indeed, $26.7 \%$ of the contests ended prior to the proposed closing time. As a result, solvers may lose their opportunity for the reward by delaying submissions. Second, since many ideation contests are simultaneously held, solvers are better off completing logo designs in one contest and then shifting to the next one. Delaying makes it more difficult for solvers to keep track of all contests in which they are participating. Therefore, Elapsed Time is a valid measure of solver effort.

${ }^{14}$ The seeker's decision on feedback volume in the first stage is not reported due to space constraints. But it is available upon request.

${ }^{15}$ Source: http://en.zhubajie.com/help/index_f_1_r_1 (accessed date July 1, 2019).

\section{References}

Aoyagi, M. 2010. Information feedback in a dynamic tournament. Games Econ. Behav. 70(2): 242-260.

Ashford, S. J., L. L. Cummings. 1983. Feedback as an individual resource: Personal strategies of creating information. Org. Behav. Hum. Decis. Process 32(3): 370-398.

Ashford, S. J., A. S. Tsui. 1991. Self-regulation for managerial effectiveness: The role of active feedback seeking. Acad. Manag. J. 34(2): 251-280.

Ashford, S. J., R. Blatt, D. VandeWalle. 2003. Reflections on the looking glass: A review of research on feedback-seeking behavior in organizations. J. Manage. 29(6): 773-799.

Bayus, B. L. 2013. Crowdsourcing new product ideas over time: An analysis of the Dell IdeaStorm Community. Management Sci. 59(1): 226-244.

Bockstedt, J., C. Druehl, A. Mishra. 2016. Heterogeneous submission behavior and its implications for success in innovation contests with public submissions. Prod. Oper. Manag. 25(7): 1157-1176.

Boudreau, K. J., K. R. Lakhani. 2013. Using the crowd as an innovation partner. Harv. Bus. Rev. 57(5): 843-863.

Boudreau, K. J., N. Lacetera, K. R. Lakhani. 2011. Incentives and problem uncertainty in innovation contests: An empirical analysis. Management Sci. 57(5): 843-863. 
Burroughs, J. E., D. W. Dahl, C. P. Moreau, A. Chattopadhyay, G. J. Gorn. 2011. Facilitating and rewarding creativity during new product development. J. Market. 75(4): 53-67.

Chan, K. W., S. Y. Li, J. J. Zhu. 2015. Fostering customer ideation in crowdsourcing community: The role of peer-to-peer and peer-to-firm interactions. J. Interact. Market. 31: 42-62.

Chen, H., N. Lim. 2013. Should managers use team-based contests? Management Sci. 59(12): 2823-2836.

Chen, H., N. Lim. 2017. How does team composition affect effort in contests? A theoretical and experimental analysis. J. Mark. Res. 54(1): 44-60.

Chen, H., S. H. Ham, N. Lim. 2011. Designing multiperson tournaments with asymmetric contestants: An experimental study. Management Sci. 57(5): 864-883.

Ederer, F. 2010. Feedback and motivation in dynamic tournaments. J. Econ. Manage. Strat. 19(3): 733-769.

Gandini, A. 2016. Digital work: Self-branding and social capital in the freelance knowledge economy. Market. Theory 16(1): 123-141.

Greene, W. H. 2000. Econometric Analysis, 6th edn. Prentice Hall, New York, NY.

Gupta, D., J. Jiang, Y. Xie, D. Chakravarti. 2019. Incentivizing user-generated content: A double-edged sword-Evidence from a natural experiment and a lab study. Working paper, Virginia Tech, Blacksburg, VA.

Hofstetter, R., J. Z. Zhang, A. Herrmann. 2018. Successive open innovation contests and incentives: Winner-take-all or multiple prizes? J. Prod. Innov. Manag. 35(4): 492-517.

Howe, J. 2006. The rise of crowdsourcing. Wired Magazine. 14(6): $1-4$.

Jiang, Y., J. Ni, X. J. Chen. 2018. Is this just about now? Short-run versus long-run rewards in crowdsourcing markets. Working paper, Johns Hopkins University, Baltimore, MD.

Kalra, A., M. Shi. 2001. Designing optimal sales contests: A theoretical perspective. Market. Sci. 20(2): 170-193.

Kerzner, H. 1995. Project Management: A Systems Approach to Planning, Scheduling, and Controlling. Van Nostrand-Reinhold, New York.

Lim, N. 2010. Social loss aversion and optimal contest design. J. Mark. Res. 47(4): 777-787.

Lim, N., M. J. Ahearne, S. H. Ham. 2009. Designing sales contests: Does the prize structure matter? J. Mark. Res. 46(3): 356-371.

Liu, T. X., J. Yang, L. A. Adamic, Y. Chen. 2014. Crowdsourcing with all-pay auctions: A field experiment on Taskcn. Management Sci. 60(8): 2020-2037.

Luo, L., O. Toubia. 2015. Improving online idea generation platforms and customizing the task structure on the basis of consumers' domain-specific knowledge. J. Market. 79(5): 100-114.

Mo, J., S. Sarkar, S. Menon. 2018. Know when to run: Recommendations in crowdsourcing contests. MIS Q. 42(3): 919-944.

Moldovanu, B., A. Sela. 2001. The optimal allocation of rewards in contests. Am. Econ. Rev. 91(3): 542-558.
Poetz, M. K., M. Schreier. 2012. The value of crowdsourcing: Can users really compete with professionals in generating new product ideas? J. Prod. Innov. Manag. 29(2): 245-256.

Prpić, J., P. P. Shukla, J. H. Kietzmann, I. P. McCarthy. 2015. How to work a crowd: Developing crowd capital through crowdsourcing. Bus. Horiz. 58(1): 77-85.

Rathi, A. 2014. To encourage innovation, make it a competition. Available at https://hbr.org/2014/11/to-encourage-innova tion-make-it-a-competition (accessed date July 1, 2019).

Rossiter, J. R., G. L. Lilien. 1994. New "brainstorming” principles. Aust. J. Manag. 19(1): 61-72.

Slot, J. H. 2013. Crossing Boundaries: Involving External Parties in Innovation. CentER, Tilburg.

Stephen, A. T., P. P. Zubcsek, J. Goldenberg. 2016. Lower connectivity is better: The effects of network structure on redundancy of ideas and customer innovativeness in interdependent ideation tasks. J. Mark. Res. 53(2): 263-279.

Terwiesch, C., K. T. Ulrich. 2009. Innovation Tournaments: Creating and Selecting Exceptional Opportunities. Harvard Business School Press, Boston, MA.

Terwiesch, C., Y. Xu. 2008. Innovation contests, open innovation, and multi-agent problem solving. Management Sci. 54(9): 1529-1543.

Toubia, O. 2006. Idea generation, creativity, and incentives. Market. Sci. 25(5): 411-425.

Toubia, O., L. Florés. 2007. Adaptive idea screening using consumers. Market. Sci. 26(3): 342-360.

Winch, C. 2010. Dimensions of Expertise: A Conceptual Exploration of Vocational Knowledge. Continuum, London.

Wooten, J. O., K. T. Ulrich. 2015. The impact of visibility in innovation tournaments: Evidence from field experiments. Working paper, University of South Carolina, Columbia, SC.

Wooten, J. O., K. T. Ulrich. 2017. Idea generation and the role of feedback: Evidence from field experiments with innovation tournaments. Prod. Oper. Manag. 26(1): 80-99.

Yoganarasimhan, H. 2013. The value of reputation in an online freelance marketplace. Market. Sci. 32(6): 860-891.

Yoganarasimhan, H. 2015. Estimation of beauty contest auctions. Market. Sci. 35(1): 27-54.

Zhang, J. 2016. Deadlines in product development. Management Sci. 62(11): 3310-3326.

\section{Supporting Information}

Additional supporting information may be found online in the Supporting Information section at the end of the article.

Appendix A: Additional Tables.

Appendix B: Proof of Propositions.

Appendix C: Additional Analysis. 